\title{
Time-Dependent Impurity in Ultracold Fermions: Orthogonality Catastrophe and Beyond
}

\author{
Michael Knap, ${ }^{1,2}$ Aditya Shashi, ${ }^{3}$ Yusuke Nishida, ${ }^{4}$ Adilet Imambekov, ${ }^{3}$ Dmitry A. Abanin, ${ }^{2}$ and Eugene Demler ${ }^{2}$ \\ ${ }^{1}$ Institute of Theoretical and Computational Physics, Graz University of Technology, 8010 Graz, Austria \\ ${ }^{2}$ Department of Physics, Harvard University, Cambridge, Massachusetts 02138, USA \\ ${ }^{3}$ Department of Physics and Astronomy, Rice University, Houston, Texas 77005, USA \\ ${ }^{4}$ Theoretical Division, Los Alamos National Laboratory, Los Alamos, New Mexico 87545, USA
}

(Received 2 July 2012; published 27 December 2012)

\begin{abstract}
The recent experimental realization of strongly imbalanced mixtures of ultracold atoms opens new possibilities for studying impurity dynamics in a controlled setting. In this paper, we discuss how the techniques of atomic physics can be used to explore new regimes and manifestations of Anderson's orthogonality catastrophe (OC), which could not be accessed in solid-state systems. Specifically, we consider a system of impurity atoms, localized by a strong optical-lattice potential, immersed in a sea of itinerant Fermi atoms. We point out that the Ramsey-interference-type experiments with the impurity atoms allow one to study the $\mathrm{OC}$ in the time domain, while radio-frequency (RF) spectroscopy probes the $\mathrm{OC}$ in the frequency domain. The $\mathrm{OC}$ in such systems is universal, not only in the long-time limit, but also for all times and is determined fully by the impurity-scattering length and the Fermi wave vector of the itinerant fermions. We calculate the universal Ramsey response and RF-absorption spectra. In addition to the standard power-law contributions, which correspond to the excitation of multiple particle-hole pairs near the Fermi surface, we identify a novel, important contribution to the OC that comes from exciting one extra particle from the bottom of the itinerant band. This contribution gives rise to a nonanalytic feature in the RF-absorption spectra, which shows a nontrivial dependence on the scattering length, and evolves into a true power-law singularity with the universal exponent $1 / 4$ at the unitarity. We extend our discussion to spin-echo-type experiments, and show that they probe more complicated nonequilibirum dynamics of the Fermi gas in processes in which an impurity switches between states with different interaction strength several times; such processes play an important role in the Kondo problem, but remained out of reach in the solid-state systems. We show that, alternatively, the OC can be seen in the energy-counting statistics of the Fermi gas following a sudden quench of the impurity state. The energy distribution function, which can be measured in time-of-flight experiments, exhibits characteristic power-law singularities at low energies. Finally, systems in which the itinerant fermions have two or more hyperfine states provide an even richer playground for studying nonequilibrium impurity physics, allowing one to explore the nonequilibrium OC and even to simulate quantum transport through nanostructures. This provides a previously missing connection between cold atomic systems and mesoscopic quantum transport.
\end{abstract}

DOI: 10.1103/PhysRevX.2.041020

\section{INTRODUCTION}

Interest in nonequilibrium quantum dynamics has increased dramatically in the last few years following the experimental realizations of synthetic many-body systems with ensembles of ultracold atoms [1,2]. With ultracold atoms it is not only possible to prepare microscopic systems with desired many-body Hamiltonians but, crucially for studying dynamics, parameters of such Hamiltonians can also be changed on time scales that are much faster than intrinsic microscopic time scales. Moreover, ultracold atomic ensembles are also very well isolated from the

Published by the American Physical Society under the terms of the Creative Commons Attribution 3.0 License. Further distribution of this work must maintain attribution to the author(s) and the published article's title, journal citation, and DOI.
Subject Areas: Atomic and Molecular Physics, Mesoscopics, Strongly Correlated Materials environment, so states prepared out of equilibrium can undergo quantum evolution without relaxation or loss of coherence [3]. Finally, a rich toolbox of atomic physics makes it possible to provide a detailed characterization of many-body systems, which is crucial for describing complicated transient states resulting from nonequilibrium dynamics. Recent experimental studies addressed such questions as the relaxation of high-energy metastable states [4-6], hydrodynamic expansion of strongly interacting fermions in optical lattices [7], decoherence of split condensates [8,9], coherent superexchange-mediated spin dynamics [10], spinor dynamics [11,12], relaxation and thermalization in 1D systems [13,14], as well as interaction quenches in fermionic systems [15].

In this paper, we discuss how one can use currently available experimental tools of ultracold atoms to study the problem of the orthogonality catastrophe (OC) in 
many-body fermionic systems. The core of this problem is understanding the response of a Fermi gas to a suddenly introduced localized impurity. This question was originally considered in the context of the x-ray absorption spectra in metals [16], where it manifests itself in power-law singularities at certain frequency thresholds.

Being one of the very few known examples of a solvable nonequilibrium many-body problems, the OC also provides a conceptual framework for understanding several fundamental phenomena in solid-state physics, including the Kondo effect [17], resonant tunneling in mesoscopic structures [18-23], 1D quantum physics beyond the Luttinger-liquid paradigm [24-26], and the motion of a heavy particle in a Fermi gas [27]. For a recent review of the OC, see Ref. [28].

The basic setup that we focus on is shown in Fig. 1. We consider a quantum-degenerate mixture of two types of atoms, e.g., a Bose-Fermi mixture [29-36] or a FermiFermi mixture [37-43]. We assume that one type of atom, called the impurity below, has a much lower density than the other. The majority atoms, referred to as host atoms, are always taken to be fermionic. The two types of atoms can have very different polarizability; hence, it is possible to create an optical lattice that strongly localizes the impurity atoms while having very little effect on the host fermions. The hyperfine spin states $|\uparrow\rangle,|\downarrow\rangle$ of the impurity atoms can be manipulated using radio-frequency (RF) fields, which allows one to switch between strongly

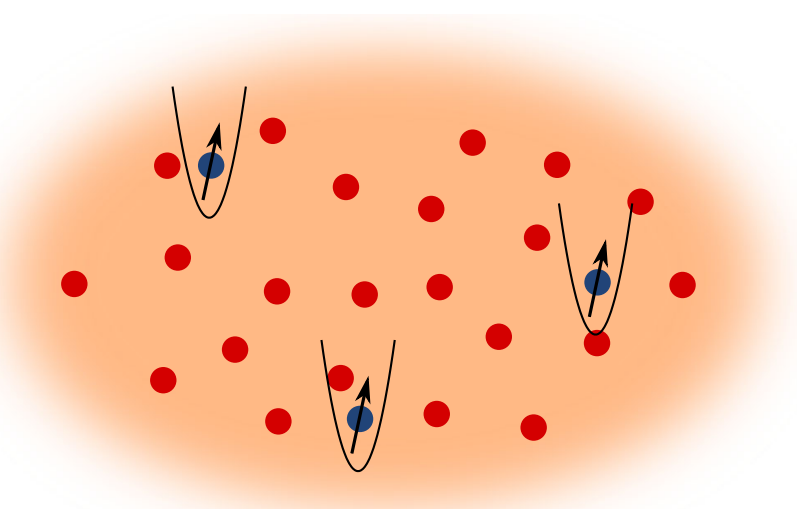

FIG. 1. Proposed experimental setup: Impurity atoms (blue dots with arrows, indicating internal states) are immersed in a sea of itinerant host fermions (red dots). The optical lattice, indicated by the black parabolas, localizes the impurity atoms without affecting the itinerant host fermions. We assume a low concentration of impurity atoms, so that we can consider scattering on a single impurity. The scattering of fermions can be controlled by applying magnetic fields and by manipulating the internal impurity states with RF pulses. The system's parameters can be controlled quickly (compared to the intrinsic time scales of the many-body system of host fermions), which makes this setup ideal for studying nonequilibrium impurity physics, including the new regimes of the OC. and weakly interacting states with respect to the host fermions, and thus introduce time-dependent impurities in the Fermi gas [44]. The OC physics generally takes place when the two hyperfine states scatter the host fermions differently. Below, for simplicity, we will assume that the $|\uparrow\rangle$ state interacts with the host fermions via a contact potential, while the $|\downarrow\rangle$ state is noninteracting. This can be described by the Hamiltonian,

$$
\hat{H}=\hat{H}_{0}+|\uparrow\rangle\langle\uparrow| \otimes \hat{V}
$$

where $\hat{H}_{0}=\sum_{k} \varepsilon_{k} c_{k}^{\dagger} c_{k}$ is the Hamiltonian of host fermions and $\hat{V}=V_{0} \sum_{k, q} c_{k}^{\dagger} c_{q}$ describes scattering. Equation (1) should be understood as having an ultraviolet cutoff $\Lambda$. The interaction strength $V_{0}$ and cutoff $\Lambda$ need to be chosen in such a way that they give the correct scattering length $a$ [45]. In our analysis below, we will present all results in terms of the scattering length $a$. Importantly, the strength of interactions between host atoms and a given hyperfine state of the impurities can be controlled by tuning the magnetic field $[44,46-49]$. We consider a regime where the density of the impurity atoms is so low that scattering processes taking place on different impurities can be analyzed separately. Thus, in the rest of the paper we will discuss the dynamics of a single impurity interacting with a Fermi gas [50].

The setup proposed above, with its tunability and precise control, provides a way to study new regimes and manifestations of the OC, which have remained out of reach in solid-state systems. First, we will show that the OC is a fully universal function of the Fermi wave vector and the impurity-host-fermion scattering length; this function will be calculated below. The full solution reveals new singularities in the RF-absorption spectra, which emerge away from the absorption threshold. Second, we will demonstrate that the Ramsey and spin-echo spectroscopystandard tools used in cold atoms-provide direct access to the $\mathrm{OC}$, as well as to more complex nonequilibrium response functions, in the time domain. Third, manifestations of the OC in the energy-counting statistics will be discussed. Finally, by generalizing the setup described above, it is possible to study the OC in the regime when the Fermi gas is driven out of equilibrium, as well as to simulate quantum transport.

Given the long illustrious history of the original problem (see Refs. [18-23,51-57]), it is useful to start by summarizing the new aspects of the OC in cold atomic systems.

Extended universality.-Experiments with ultracold atoms can demonstrate the universality of the $\mathrm{OC}$ in a much broader sense than was previously discussed for electron systems. The most basic quantity demonstrating the $\mathrm{OC}$ is the time-dependent overlap function,

$$
S(t)=\left\langle\psi_{0}\left|e^{i \hat{H}_{i} t / \hbar} e^{-i \hat{H}_{f} t / \hbar}\right| \psi_{0}\right\rangle
$$

where $\left|\psi_{0}\right\rangle$ is the initial ground state of the Fermi gas, and $\hat{H}_{i(f)}$ is the Hamiltonian before (after) the impurity is 
switched on. In solid-state systems, the universality was understood only as a statement about the long-time behavior of $S(t)$. At long times, $t \gg \hbar / E_{F}, E_{F}$ being the Fermi energy, the overlap exhibits a power-law decay [52], reflecting Anderson's OC [53,54]. The power-law decay is the result of the large phase space available for exciting multiple low-energy particle-hole pairs that are created when the impurity potential is switched on.

Although the OC has been considered as an "exactly solvable" dynamical many-body problem, in solid-state systems model parameters either are not known accurately or provide a crude approximation to much more complicated many-body processes. For example, a local impurity potential comes from the Coulomb potential of a hole screened by the conduction electrons. A one-particlescattering picture commonly used in the analysis of the OC [51-53], which neglects many-body aspects of electron-electron interactions, is only an approximation valid for low-energy scattering processes of electrons close to the Fermi energy. Furthermore, the density of states for the conduction-band electrons is typically not known and can be modified by the electron interactions. In addition, extrinsic degrees of freedom, including phonons, can play an important role in the OC dynamics leading to extra decay factors. Hence, one can neither calculate the full time dependence of $S(t)$ nor claim its universality at all time scales.

By contrast, in the case of ultracold atoms, one can create a gas of Fermi atoms, which are well isolated, do not interact with each other, and have a simple parabolic dispersion. The interaction of the localized impurity atoms and conduction-band fermions is fully characterized by a single parameter, the scattering length $a$. In the case of wide resonances, one finds a universal behavior of the energy dependence of the scattering amplitude,

$$
\frac{1}{f(E)}=-\frac{1}{a}+i \frac{\sqrt{2 m E}}{\hbar}
$$

where $E$ is the energy of the scattering fermion [58]. Such scattering amplitude leads to the following universal dependence of the scattering phase shift on the momentum of the scattered fermion:

$$
\delta(k)=-\tan ^{-1} k a,
$$

where $-\pi / 2<\delta(k)<\pi / 2$. Consequently, the entire function $S(t)$ (and not only its long-time asymptote) is a universal function of $k_{F} a$ and $E_{F} t$ ( $k_{F}$ is the Fermi wave vector and $E_{F}$ is the Fermi energy),

$$
S(t)=S\left(k_{F} a, E_{F} t / \hbar\right) .
$$

The main result of this work is the calculation of universal functions $S(t)$, as well as more complicated time-domain response functions. Specific examples are shown in Figs. 4, 6, and 7.
Measuring orthogonality catastrophe in the time domain.-In solid-state systems, the $\mathrm{OC}$ as characterized by the overlap function in Eq. (2) is observed indirectly in the frequency dependence of the absorption spectrum, given by

$$
A(\omega)=\frac{1}{\pi} \operatorname{Re} \int_{0}^{\infty} e^{i \omega t} S(t) d t .
$$

The power-law decay of the overlap function in the time domain translates into power-law threshold singularities in the absorption spectra $[16,52,54]$. In ultracold atoms, one can perform similar measurements of the OC in the frequency domain by doing RF spectroscopy on impurity atoms. Examples of the universal absorption spectra for different values of $k_{F} a$ are shown in Fig. 2.

However, it may be more illuminating to measure $S(t)$ in the time domain using Ramsey-type interference, which is a well-established experimental technique in atomic physics. While it was originally designed for metrology applications, it has been realized recently that it can also be used as a probe of many-body dynamics $[8,61-65]$.

The idea of the proposed experiment is as follows (also see Ref. [66]): Initially, the impurity is prepared in the down state $|\downarrow\rangle$, and the fermions are in the ground state $\left|\psi_{0}\right\rangle$. Then, the Ramsey interferometry is performed: At time $t=0$, a $\pi / 2$ pulse is applied, such that the system is driven into the superposition state $\frac{|\downarrow\rangle+|\dagger\rangle}{\sqrt{2}} \otimes\left|\psi_{0}\right\rangle$. The two states evolve differently since the $|\uparrow\rangle$ and $|\downarrow\rangle$ states interact differently with the Fermi sea:

$|\Psi(t)\rangle=\frac{1}{\sqrt{2}}|\downarrow\rangle \otimes e^{-i \hat{H}_{i} t / \hbar}\left|\psi_{0}\right\rangle+\frac{1}{\sqrt{2}}|\uparrow\rangle \otimes e^{-i \hat{H}_{f} t / \hbar}\left|\psi_{0}\right\rangle$.

The fermions stay undisturbed in the first state, while the impurity scattering excites multiple particle-hole pairs in the second state. Performing a second $\pi / 2$ pulse after time $t$ and measuring $\left\langle\Psi(t)\left|\hat{S}_{x}\right| \Psi(t)\right\rangle$ gives

$$
\left\langle\hat{S}_{x}(t)\right\rangle=\operatorname{Re}\left\langle\psi_{0}\left|e^{i \hat{H}_{i} t / \hbar} e^{-i \hat{H}_{f} t / \hbar}\right| \psi_{0}\right\rangle=\operatorname{Re} S(t) .
$$

In the equation above, we neglected the trivial phase factor arising from the energy difference of states $|\uparrow\rangle$ and $|\downarrow\rangle$. Thus, the Ramsey interferometry provides a direct measurement of the OC overlap [67].

The basic Ramsey-type experiment described above corresponds to a local quench-type dynamics in which the impurity strength is changed once. One can also use the Hahn spin echo, as well as more complicated spin-echo-type sequences, to realize processes in which the impurity strength effectively switches between different values multiple times. As we show below, the response of the Fermi gas to such processes is characterized by a power-law decay of the overlap, with an exponent enhanced compared to the case of the usual OC.

The predicted faster decay of the spin-echo response may seem counterintuitive at first glance because it goes 


\section{(a)}
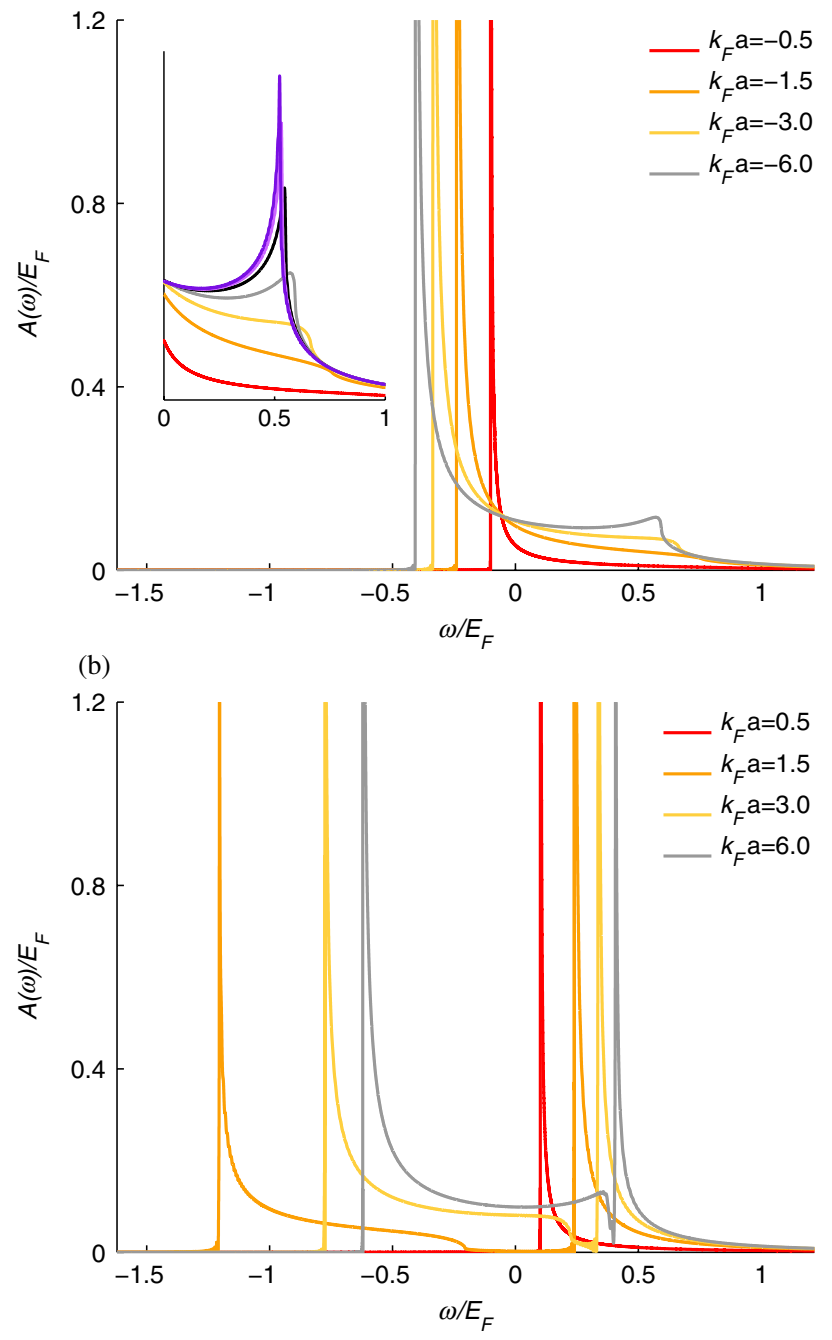

FIG. 2. RF-absorption spectra for different values of scattering length $a$. (a) When $a<0$, no bound state is present and the spectrum exhibits one power-law edge singularity at $\hbar \omega_{0}=\Delta E$ due to the processes illustrated in Fig. 3(b). In addition, a weak nonanalyticity at $\hbar \omega_{1}=\Delta E+E_{F}$ is found, attributed to the excitations from the bottom of the band, illustrated in Fig. 3(c). This nonanalyticity is magnified and plotted for additional large values of $k_{F} a=-12,-18,-24$ in the inset in (a). At unitarity, the nonanalyticity transforms into a true divergence with exponent $1 / 4$, which is described by a universal result shown in Eq. (18). (b) When $a>0$, the impurity creates a bound state. The spectra in this case exhibit two threshold singularities, which are offset by an energy $\left|E_{F}-E_{b}\right|$, as well as an additional cusplike singularity, the origin of which is illustrated in Fig. 3(f).

against the atomic-physics intuition; it stems from the fact that the spin-echo sequence does not "undo" the evolution of the Fermi gas under impurity scattering, but instead creates a state in which the impurity pseudospin and Fermi gas are strongly entangled [68]. From the experimental point of view, spin-echo experiments should have an additional advantage in that they allow one to cancel slow fluctuations of the magnetic field.
New universal features: Bottom of the band physics.What are the new universal characteristics that one can find in $S(t)$, as well as in the corresponding absorption spectra $A(\omega)$ at intermediate time scales? One feature that can be seen from Fig. 4 is the oscillations of $S(t)$ on the time scale $\hbar E_{F}^{-1}$. The origin of this novel feature is the following: After the impurity is introduced, there is a class of processes in which, in addition to a large number of lowenergy particle-hole pairs, an extra hole with energy of approximately $E_{F}$ is excited near the bottom of the band. The phase space of such processes is enhanced, owing to the van Hove singularity in the density of states at the band bottom in one dimension (note that the $\mathrm{OC}$ is essentially a 1D problem since only the $s$-wave channel is important). The combined dynamics of the high-energy hole and the low-energy particle-hole pairs result in an additional weaker power-law contribution to the overlap function.

This contribution to the overlap function becomes even more evident in the frequency domain, where it gives rise to a cusp singularity at the energy $E_{F}$ above the threshold (see Fig. 2). We find that, as the Feshbach resonance is approached from the side of the negative scattering length, the cusp develops into a true singularity with a universal exponent $1 / 4$; for any finite value of $k_{F} a$, this peak is smeared, but its overall shape is described by a universal function, which is discussed below. We note that such behavior of the response function across the Feshbach resonance is rather unusual, since it only shows a truly singular behavior exactly at the resonance. For comparison, in conventional studies of the crossover from Bardeen-Cooper-Schrieffer superfluidity to Bose-Einstein condensation [70], most of the measured quantities show a smooth behavior exactly at the resonance.

Nonequilibrium orthogonality catastrophe.-So far we assumed that the itinerant host fermions do not have any internal degrees of freedom. A generalization of the setup shown in Fig. 1 to the case of a multicomponent Fermi gas (with multiple internal states) allows one to realize a wide range of dynamical impurity phenomena in nonequilibrium Fermi gases. This is an even richer class of problems that arises in mesoscopics [28]; in particular, problems of this kind describe the quantum transport through any mesoscopic structure (e.g., a point contact), where the Fermi seas in two or more leads are kept at different chemical potentials. Mathematically, such problems can be reduced to a non-Abelian Riemann-Hilbert problem [20], the solution of which is not known in a general case.

While transport is usually difficult to study in systems of ultracold atoms (see, however, [71]), we will show that the extension of our setup provides a way of simulating the quantum transport. More generally, it allows one to study the response of the nonequilibrium Fermi gas in a controlled setting.

New quantum observables and distribution functions.OC experiments with ultracold atoms should make it possible to study quantum variables that are not accessible in 
electron systems. For example, the full energy of an interacting many-body system can be measured [48,72-74]. Moreover, it is possible to measure not only the average values but also fluctuations of quantum observables [75], and in some cases even the full distribution functions $[65,76]$.

In the quantum-impurity system as in Fig. 1, following the impurity-potential quench, the system is no longer in an energy eigenstate and the full distribution function of the total energy should also exhibit power-law-type singularities (see Refs. [20,77,78] and discussion below), which provides an alternative manifestation of the OC.

Full-counting statistics of scattering processes should provide an intriguing connection to extensive theoretical research in mesoscopic physics [28]. It is worth noting that the measurements of charge-counting statistics were notoriously difficult in solid-state systems [79]. Given the available experimental tools, such measurements should become possible in cold atomic systems, with an additional advantage that the full-counting statistics of scattering events can be measured for fermions in specific energy windows rather than in the whole energy range.

This paper is organized as follows: In Sec. II we describe our approach to the $\mathrm{OC}$, and present the results for the universal overlap function $S(t)$, which can be measured by the Ramsey interferometry. The universal RF spectra $A(\omega)$ are evaluated, and their properties, singularities, as well as the novel singular feature appearing at $E_{F}$ above the threshold are discussed. Extensions to the Hahn spin echo and more complicated spin-echo-type experiments, in which effectively the impurity strength changes several times, are studied in Sec. III. Also, in Sec. III we study the experimentally relevant effect of finite temperature on the Ramsey and spin-echo responses. The manifestations of the $\mathrm{OC}$ in the energy-counting statistics and generalizations to the nonequilibrium OC using multicomponent host atoms are discussed in Secs. IV and V, respectively. Finally, we connect the proposed setup to existing cold-atom experiments and conclude our findings in Sec. VI.

\section{UNIVERSAL ORTHOGONALITY CATASTROPHE}

In this section, our main goal will be to calculate the universal response functions, $S(t)$ and $A(\omega)$. To simplify the discussion we assume that the $|\downarrow\rangle$ state of the impurity does not interact with the host fermions and only the $|\uparrow\rangle$ state gives rise to scattering. A generalization of our analysis to the case when both $|\uparrow\rangle$ and $|\downarrow\rangle$ states interact with the fermions is straightforward. Assuming that the impurity atom is in the rotationally symmetric ground state of the confining parabolic potential and cannot be excited to higher states (the energy of the confining potential is much larger than the Fermi energy), we only need to consider the $s$-wave scattering of host atoms on the impurity. The corresponding Hamiltonian can be written in the form of Eq. (1). The Ramsey sequence described above measures the real part of the overlap function, see Eq. (8), where $\hat{H}_{i}=\hat{H}_{0}$ and $\hat{H}_{f}=\hat{H}_{0}+\hat{V}$.

There are two physically distinct cases for which the asymptotic behavior of the overlap function (as well as the shape of the absorption spectrum) is qualitatively different [55]: (i) $a<0$, when the impurity potential does not create a bound state, and (ii) $a>0$, when there is a bound state with energy

$$
E_{b}=-\frac{\hbar^{2}}{2 m a^{2}} .
$$

To gain an intuition about the behavior of the overlap function $S(t)$ in the two cases, it is convenient to rewrite it by inserting a complete set of eigenstates $\left\{\psi_{\alpha}\right\}$ (with corresponding eigenenergies $\left\{E_{\alpha}\right\}$ ) of the Hamiltonian $\hat{H}_{0}+\hat{V}$ into Eq. (2):

$$
S(t)=\sum_{\alpha}\left|\left\langle\psi_{0} \mid \psi_{\alpha}\right\rangle\right|^{2} e^{i\left(E_{0}-E_{\alpha}\right) t / \hbar} .
$$

Explicit expressions for $\psi_{\alpha}$ and $E_{\alpha}$ are given in Appendix A. In Fig. 3, we illustrate the dominant intermediate states $\left|\psi_{\alpha}\right\rangle$ giving rise to the power-law contributions to the overlap. In the case $a<0$, the main contribution to $S(t)$ is due to the intermediate states $\left|\psi_{\alpha}\right\rangle$ in which multiple low-energy particle-hole pairs are created near the Fermi surface [see Fig. 3(b)]. The large phase space of such excitations gives rise to the power-law decay of $S(t)$ at long times; see Eq. (12). In the case $a>0$, when there is a bound state, there are two distinct important sets of states $\left|\psi_{\alpha}\right\rangle$, which involve many excitations near the Fermi surface, but differ by the bound state being either empty or filled [Figs. 3(d) and 3(e)]. The contributions of these two sets of states into $S(t)$ separate, such that at long times $S(t)$ is given by the sum of two power laws with different exponents; see Eq. (15).

Below we identify another important set of intermediate states, which gives rise to an additional weaker power-law contribution to the overlap function. Such states involve a single hole excited from the vicinity of the band bottom, in addition to a number of low-energy particle-hole pairs [Figs. 3(c) and 3(f)]; the contribution of these states to the overlap function is enhanced by the van Hove singularity at the bottom of the $1 \mathrm{D}$ band. As we shall see below, this new contribution results in an unusual kink feature in the absorption spectrum, which at the unitarity $\left(\left|k_{F} a\right|=\infty\right)$ develops into a full power-law singularity with a universal exponent $1 / 4$.

\section{A. Method}

Our analysis of the universal OC is based on the representation of the response functions in terms of functional determinants $[20,21,55,80]$. We are interested in evaluating many-body averages of products of exponents of operators quadratic in creation and annihilation operators [see Eqs. (1) and (8)]. Such many-body quantities can be reduced to 

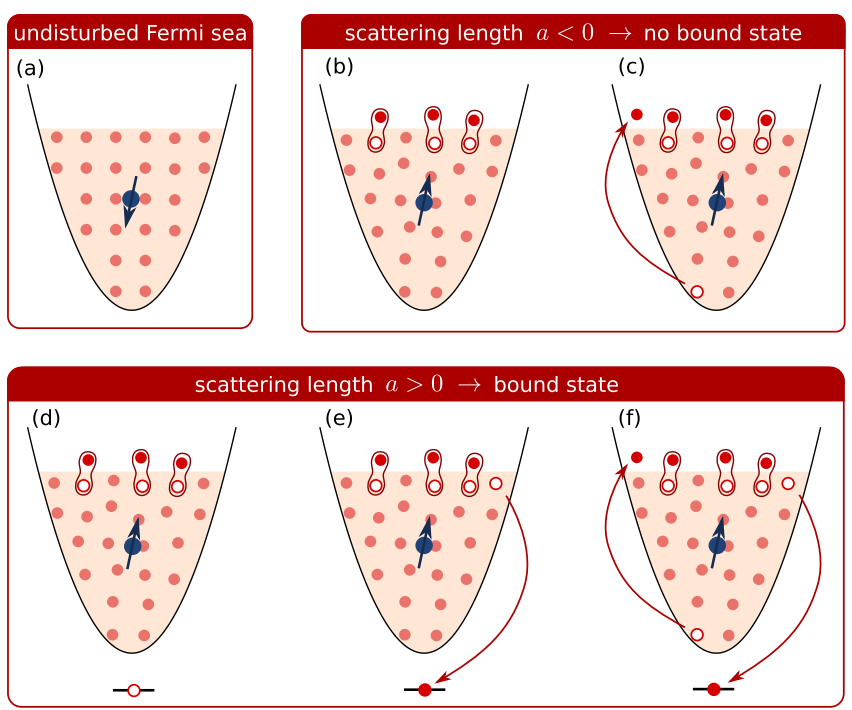

FIG. 3. Schematic illustration of the intermediate excited sets of states giving power-law contributions to the overlap function, which result in the threshold singularities in the RF-absorption spectrum. (a) Undisturbed Fermi sea (impurity is in the $|\downarrow\rangle$ state). (b)-(c) Case $a<0$ (no bound state): (b) Multiple low-energy particle-hole pairs, giving rise to the leading power-law decay, Eq. (12), of the overlap; (c) an additional particle is promoted from the bottom of the band to the vicinity of the Fermi surface. This contribution leads to the oscillations with period $2 \pi \hbar / E_{F}$ in the overlap function [see Fig. 4(a)], as well as to the new cusplike singularity in the absorption spectra [Fig. 2(a)]. (d)-(f) For the case $a>0$, when the impurity potential creates a bound state, there are three important sets of states: (d) The bound state is empty and multiple low-energy excitations are created, (e) the bound state is filled, and (f) an additional particle is excited from the bottom of the band to the Fermi surface. The processes, (d) and (e), lead to the behavior, Eq. (15), of the overlap function while (f) leads to a faster-decaying oscillating contribution. The three contributions result in the absorption spectra shown in Fig. 2(b).

functional determinants in the space of single-particle orbitals. The overlap $S(t)$, in particular, can be written in the following form $[20,21,80]$ :

$$
S(t)=\operatorname{det}[1-\hat{n}+\hat{R}(t) \hat{n}], \quad \hat{R}(t)=e^{i \hat{h}_{0} t / \hbar} e^{-i\left(\hat{h}_{0}+\hat{v}\right) t / \hbar},
$$

where $\hat{n}$ is the occupation number operator, $\hat{n}|\varepsilon\rangle=n(\varepsilon)|\varepsilon\rangle$; at finite temperature, $n(\varepsilon)$ is given by the Fermi distribution, $n(\varepsilon)=\frac{1}{\exp [(\varepsilon-\mu) / T]+1}$. The operators $\hat{h}_{0}, \hat{h}_{0}+\hat{v}$ are the single-particle Hamiltonians in the absence and presence of impurity, respectively.

While previous work $[20-22,81]$ concentrated on analyzing the asymptotic behavior (at times $t \gg \hbar / E_{F}$ ) of the functional determinant of the type shown in Eq. (11), here our goal is to find the response functions at all times. Therefore, we numerically evaluate the functional determinants.
We consider the case of a finite system confined in a ball. This allows us to view the operator in Eq. (11) as a finite-dimensional matrix for the case of zero temperature, when $n(\varepsilon)$ is nonzero only for a finite number of states under the Fermi level. It turns out that the matrix elements of the matrix $\hat{R}(t)$ can be evaluated to high precision by appropriately truncating the (infinite dimensional) matrix $e^{-i\left(\hat{h}_{0}+\hat{v}\right) t}$. Taking the finite-size scaling, we obtain the universal behavior of the overlap in the continuum limit. The details of our discretization and truncation procedures can be found in Appendix A. An advantage of our approach is that it can be easily generalized to other geometries, including the experimentally important case of the harmonic trap. Moreover, below we extend our approach to include finite-temperature effects, which will certainly play an important role in experiments (see Sec. III).

The structure of the single-particle Hilbert space depends on the sign of the scattering length: When $a<0$, the eigenstates of the Hamiltonian $\hat{h}_{0}+\hat{v}$ are scattering states; when $a>0$, the spectrum should be supplemented by the bound state. This translates into a different asymptotic behavior of the overlap function $S(t)$ and the absorption function $A(\omega)$, as discussed below.

\section{B. Universal overlap functions}

The absolute value of the resulting universal function $S(t)$ in the two cases is shown in Fig. 4. [We note that the quantity $\operatorname{Re} S(t)$, measured in the Ramsey experiment, is quickly oscillating with a period set by the energy difference between two hyperfine states as well as by the difference of the ground state energies of the Hamiltonians $\hat{H}_{0}$ and $\hat{H}_{0}+\hat{V}$; when plotting the Ramsey response in Fig. 4, we have ignored this trivial phase and illustrated the Ramsey amplitude $|S(t)|$.]

In the case $a<0$, see Fig. 4(a), the long-time asymptotic behavior at $t \gg \hbar / E_{F}$ agrees with the analytic result [52],

$$
S(t) \approx C e^{-i \Delta E t / \hbar}\left(\frac{1}{i E_{F} t / \hbar+0^{+}}\right)^{\alpha}, \quad \alpha=\frac{\delta_{F}^{2}}{\pi^{2}},
$$

where

$$
\delta_{F}=-\tan ^{-1}\left(k_{F} a\right)
$$

is the scattering phase shift at the Fermi wave vector, and

$$
\Delta E=-\int_{0}^{E_{F}} \frac{d E}{\pi} \delta(\sqrt{2 m E})
$$

is the energy renormalization of the Fermi sea due to the impurity level [82]. The power law in Eq. (12) is a manifestation of the "shakeup" process after the sudden switching of the impurity potential, which creates multiple low-energy particle-hole excitations near the Fermi surface; see Fig. 3(b). The dependence of the prefactor $C$ on $k_{F} a$, calculated from fitting the numerical results at long 


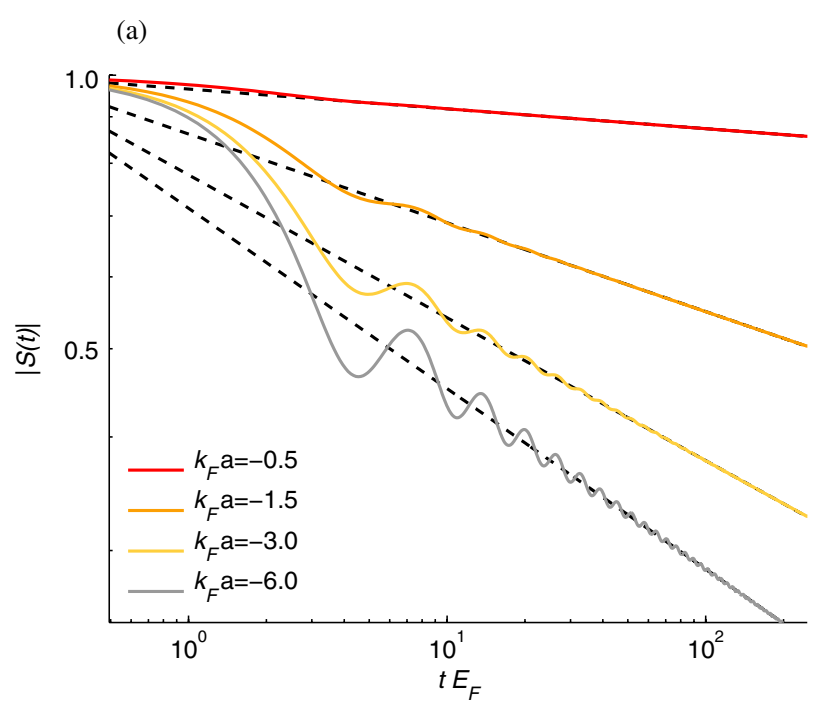

(b)

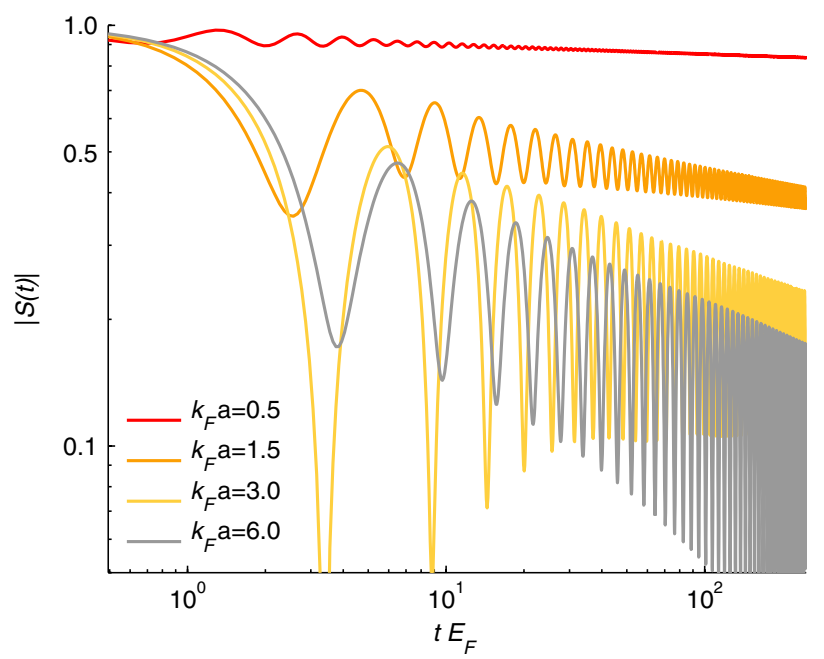

FIG. 4. Universal overlap function $|S(t)|$, which can be measured in the Ramsey interferometry experiment, is shown for different values of the scattering length $a$; see legend. Panel (a) shows $|S(t)|$ for the case $a<0$, when the impurity potential does not create a bound state. The dashed lines are power-law fits, Eq. (12), at long times. At intermediate times, oscillations coming from exciting an extra hole near the bottom of the band are visible. Panel (b) shows $|S(t)|$ for the case $a>0$, when the impurity potential creates a bound state. The asymptotic behavior is described by Eq. (15) and exhibits strong oscillations.

times with Eq. (12), is illustrated in Fig. 5. The overlap function $S(t)$ exhibits oscillations with period $2 \pi \hbar / E_{F}$ (see Fig. 4), which are due to the processes in which an extra particle is excited from the bottom of the band to the Fermi level [see Fig. 3(c)].

For the case $a>0$, see Fig. 4(b), the overlap function $S(t)$ has two main contributions coming from the intermediate states where the bound state is either filled or empty. This alters the asymptotic behavior, which is now represented by the sum of two power laws [55]:

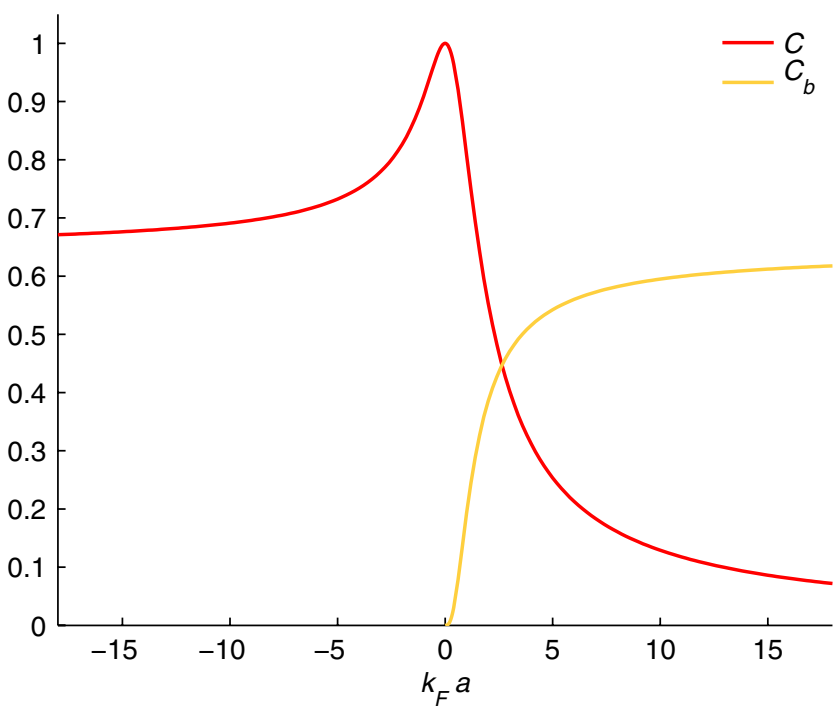

FIG. 5. The prefactors that appear in the asymptotic behavior of the overlap function, as well as in the RF response near the thresholds at $\omega_{0}, \omega_{b}$; see Eqs. (12) and (15)-(17). The prefactors were obtained from the asymptotic form of the numerically evaluated overlap functions.

$$
\begin{aligned}
S(t) \approx & C e^{-i \Delta E t / \hbar}\left(\frac{1}{i E_{F} t / \hbar+0^{+}}\right)^{\alpha} \\
& +C_{b} e^{-i\left(\Delta E-E_{F}+E_{b}\right) t / \hbar}\left(\frac{1}{i E_{F} t / \hbar+0^{+}}\right)^{\alpha_{b}}, \\
\alpha_{b}= & \left(1+\delta_{F} / \pi\right)^{2},
\end{aligned}
$$

where $C$ and $C_{b}$ are $k_{F} a$-dependent numerical constants. The strong oscillations with period $2 \pi \hbar /\left(E_{F}-E_{b}\right)$, predicted by the above formula, are evident in Fig. 4(b). Our approach allows the numerical calculation of the prefactors $C$ and $C_{b}$, yielding the result shown in Fig. 5 [83]. In addition, the overlap function exhibits faster-decaying oscillations with period $2 \pi \hbar / E_{F}$, which correspond to the processes in which an extra electron is promoted from the bottom of the band to the Fermi surface [see Fig. 3(f)]; in Fig. 4(b), these are not visible because they are masked by the stronger oscillations with period $2 \pi \hbar /\left(E_{F}-E_{b}\right)$.

It is worth noting that in experiments, the host fermions are always confined in a harmonic trap with typical level spacing $\omega_{t}$. In the above analysis, we have implicitly assumed $\omega_{t}$ to be negligibly small. We note that at short times $t \lesssim \hbar / \omega_{t}$, the harmonic confinement effectively does not play any role, and the Ramsey experiment yields the universal overlap function calculated above. At longer times, the overlap function remains universal but acquires an additional dependence on $E_{F} / \omega_{t}$, which can be calculated within our approach.

\section{Universal radio-frequency spectra}

We now use the above results for the overlap function to evaluate the RF spectra. Numerically calculating $S(t)$ for a 
very large interval of $t$ values, necessary for a precise Fourier transform, is computationally challenging. We circumvent this difficulty as follows: We choose a cutoff $t_{*} \gg$ $\hbar / E_{F}$; at times $t<t_{*}$, function $S(t)$ is evaluated numerically, while at $t>t_{*}$, we match $S(t)$ to its asymptotic power-law behavior given by Eq. (12) for $a<0$ and Eq. (15) for $a>0$. Calculating the real part of the Fourier transform of the resulting function, we obtain the RF spectra.

The behavior of $A(\omega)$ for negative and positive scattering lengths is qualitatively different (see Fig. 2). The absorption spectrum for $a<0$ exhibits one edge singularity, with an exponent $1-\alpha$ that follows from Eq. (12). For $a>0$, the asymptotic behavior, Eq. (15), gives rise to a double-threshold absorption spectrum; due to the presence of the bound state the spectrum is characterized by two singularities at energies $\hbar \omega_{b}=\Delta E-E_{F}+E_{b}$ and $\hbar \omega_{0}=\Delta E$. The first threshold corresponds to filling the bound state following absorption, and the second to leaving it empty [55]. As follows from the definition of $A(\omega)$, Eq. (6), and from the asymptotic form, Eq. (15), the two threshold singularities are characterized by different exponents, $1-\alpha_{b}$ and $1-\alpha$, respectively. The excitation processes which correspond to these singularities are illustrated in Figs. 3(d) and 3(e). The response near these thresholds can be obtained using Eqs. (12) and (15), which gives

$$
\begin{gathered}
S\left(\omega-\omega_{0}\right) \approx \frac{2 \pi C \theta\left(\omega-\omega_{0}\right)}{\Gamma(\alpha)\left(E_{F} / \hbar\right)^{\alpha}}\left|\omega-\omega_{0}\right|^{\alpha-1}, \\
\hbar \omega_{0}=\Delta E, \\
S\left(\omega-\omega_{b}\right) \approx \\
\frac{2 \pi C_{b} \theta\left(\omega-\omega_{b}\right)}{\Gamma\left(\alpha_{b}\right)\left(E_{F} / \hbar\right)^{\alpha_{b}}}\left|\omega-\omega_{b}\right|^{\alpha_{b}-1}, \\
\hbar \omega_{b}=\Delta E-E_{F}+E_{b} .
\end{gathered}
$$

The most interesting new feature of the universal spectra shown in Fig. 2 is the nonanalyticity of $A(\omega)$ at frequency $\hbar \omega_{1}=\Delta E+E_{F}$ for $a<0$ and $\hbar \omega_{1}=\Delta E+E_{b}$ for $a>0$; the physical origin of this feature was already discussed above. As the unitarity is approached, the nonanalytic feature becomes more prominent. This phenomenon, as well as the full structure of $A(\omega)$ at $k_{F}|a| \gg 1$, can be understood as the result of a nontrivial interplay between two-body physics that involves the impurity and the hole near the bottom of the band, and the dynamics of multiple particle-hole excitations created at the Fermi surface. The possibility of a nontrivial interplay between many-body and few-body physics is a unique feature of ultracold-atom physics, and has attracted significant theoretical $[86,87]$ and experimental [88] interest recently.

We have developed an analytic theory of this new feature; here, we briefly summarize the essence of our approach and main results, providing the full results in Appendix B; the details of the solution will be provided in Ref. [85]. The idea is that, in the time domain, the contributions from the Fermi-surface excitations and from the single hole at the bottom of the band to the determinant, Eq. (11), factorize. The former contribution is given by the power law which can be obtained within the functional-determinant approach, while the latter can be evaluated exactly within the two-body theory [85].

In the frequency domain, the result can be written as a convolution of the two terms describing these two processes (see Appendix B); this gives rise to a cusplike singularity at an energy $\Delta E+E_{F}$ for $a<0$ and $\Delta E+E_{b}$ for $a>0$. Away from the unitarity, this singularity is smeared on the energy scale of approximately $\hbar^{2} / 2 m a^{2}$ (for either sign of the scattering length $a$ ); the origin of this smearing lies in the dynamics of the hole.

Remarkably, right at the unitarity, $\left|k_{F} a\right|=\infty, \hbar^{2} / 2 m a^{2}$ vanishes, and a full nonsmeared power-law singularity with the universal exponent $1 / 4$ develops; this singularity is asymmetric, and is described by

$$
\begin{aligned}
A(\omega) \approx & \frac{1.74\left|\omega-\omega_{1}\right|^{-1 / 4}}{\left(E_{F} / \hbar\right)^{3 / 4}} \times\left[\frac{\pi^{1 / 2} \theta\left(\omega-\omega_{1}\right)}{\Gamma(3 / 4)}\right. \\
& \left.+\pi^{-1 / 2} \Gamma(1 / 4) \theta\left(\omega_{1}-\omega\right)\right]
\end{aligned}
$$

for $\left|k_{F} a\right|=\infty$ and $\hbar\left|\omega-\omega_{1}\right| \ll E_{F}$.

When $a<0$, this peak gets smeared out at energies of the order of $\hbar^{2} / 2 m a^{2}$ from its maximal value; the evolution of the peak depending on the value of $k_{F} a$ is illustrated in the inset to Fig. 2(a).

For the case $a>0$, the true bound state with energy $E_{b}$ "pinches off" from the bottom of the band and leads to a threshold with an exponent $3 / 4$. Thus, for large but finite $k_{F} a>0$ the universal form of $A(\omega)$ near $\omega_{1}$ has a characteristic double-peak structure, as is seen in Fig. 2(b) for $k_{F} a=6.0$. For increasing interaction parameter $k_{F} a$, the novel kink feature approaches the singularity at $\omega_{0}$ where the bound state is empty, as the energy difference is only of the order of $E_{b}$, which tends to zero when the unitarity is approached.

The universal contribution due to the bottom of the band is a unique feature of ultracold spinless fermions that has no analog in conventional solid-state systems. Indeed, the universal behavior coming from the excitations in the vicinity of the Fermi surfaces is ubiquitous in solid-state systems, and is controlled by Fermi-liquid theory [89]. On the other hand, away from the Fermi surface, fermionic quasiparticles are not well defined in 3D interacting systems, and thus bottom-of-the-band contributions to the orthogonality catastrophe cannot be probed in solid-state systems. In contrast, for spinless cold atoms, such contributions are well defined, since the interactions between atoms in the $s$-wave channel are absent. Thus, fermionic excitations are well defined for all energies, including the vicinity of the band bottom. 


\section{SPIN-ECHO RESPONSE}

Now we proceed with discussing spin-echo-type sequences, which allow one to measure the response of the Fermi gas to a process in which the internal impurity states switch multiple times. The spin-echo sequences have an important advantage compared to the Ramsey interferometry in that they are not sensitive to the slowly fluctuating magnetic fields typically present in experiments.

The main effect of the fluctuating fields is to introduce an energy difference $\Delta \varepsilon$ between the $|\uparrow\rangle$ and $|\downarrow\rangle$ states. Generally, this would affect the outcome of the Ramsey experiment: Owing to the different phase picked up by the $|\uparrow\rangle$ and $|\downarrow\rangle$ states, the measured value of $\left\langle\hat{S}_{x}\right\rangle$ is given by

$$
\left\langle\hat{S}_{x}(t)\right\rangle=\frac{S(t) e^{i \Delta \varepsilon t /(2 \hbar)}+S^{*}(t) e^{-i \Delta \varepsilon t /(2 \hbar)}}{2} .
$$

The phase factor $e^{i \Delta \varepsilon t /(2 \hbar)}$ is different from shot to shot; thus, averaging over different shots gives rise to an additional decay of $\left\langle S_{x}(t)\right\rangle$. This extra decay would complicate the observation of the OC. Similarly to quantumoptics experiments, the undesired contribution of the fluctuating magnetic fields can be eliminated in the spinecho experiment.

We first consider the Hahn spin-echo protocol: Initially, the system is prepared in the state $|\downarrow\rangle \otimes\left|\psi_{0}\right\rangle$; at time $-t$ a $\pi / 2$ pulse is applied, followed by the $\pi$ pulse at $t=0$. Finally, at time $t$ another $\pi / 2$ pulse is applied. Similar to the case of the Ramsey experiment, we can show that $S_{x}$ measured after such a sequence is given by

$$
\left\langle\hat{S}_{x, 1}(t)\right\rangle=\operatorname{Re}\left[S_{1}(t)\right], \quad S_{1}(t)=\left\langle\psi_{0}\left|\hat{U}_{2}^{-1} \hat{U}_{1}\right| \psi_{0}\right\rangle,
$$

where

$$
\begin{aligned}
& \hat{U}_{1}=e^{-i \hat{H}_{0} t / \hbar} e^{-i\left(\hat{H}_{0}+\hat{V}\right) t / \hbar}, \\
& \hat{U}_{2}=e^{-i\left(\hat{H}_{0}+\hat{V}\right) t / \hbar} e^{-i \hat{H}_{0} t / \hbar},
\end{aligned}
$$

are the operators describing the evolution of the Fermi-gas state that was initially coupled to the $|\uparrow\rangle$ and $|\downarrow\rangle$ states of the impurity. This correlation function describes nontrivial Fermi-gas dynamics in a process where the impurity switches between different states several times. Similar response functions arise in the analysis of the Kondo problem [17]. To understand the behavior of the spinecho response, we represent it as a functional determinant given by Eq. (11) with $\hat{R}=\hat{u}_{2}^{-1} \hat{u}_{1}\left(\hat{u}_{1}, \hat{u}_{2}\right.$ being the singleparticle analogues of operators $\hat{U}_{1}, \hat{U}_{2}$ ). As can be shown analytically using the method of Ref. [20], the asymptotic behavior of such a determinant at long-time $t \gg \hbar / E_{F}$ is given by a power law,

$$
S_{1}(t) \propto t^{-3 \delta_{F}^{2} / \pi^{2}},
$$

with an exponent 3 times larger than that of the OC. The universal behavior of the spin-echo response for arbitrary times, calculated numerically using the determinant approach, is compared to the standard OC probed with Ramsey interference in Fig. 6.

The predicted faster decay of the spin-echo response is somewhat unexpected: The intuition from quantum optics would suggest that the spin-echo response generally eliminates the broadening due to a slowly fluctuating environment, and therefore should be characterized by the slower decay compared to the Ramsey interference. This intuition, however, relies on the assumption that the environment only affects the relative phase of the two hyperfine states; thus, the operators describing the effect of the environment on the two pseudospin states commute (and therefore the spin-echo sequence can undo the effect of the environment). The above discussion illustrates that this assumption breaks down for the case when the Fermi gas plays the role of environment: The reason is that the operators $e^{-i\left(\hat{H}_{0}+\hat{V}\right) t}$ and $e^{-i \hat{H}_{0} t}$ no longer commute, and therefore $\hat{U}_{1} \neq \hat{U}_{2}$; this results in the nontrivial form of the spinecho response, Eq. (19), and its faster decay.

The generalized spin-echo sequence, in which $(2 n-1)$ $\pi$ pulses are applied at equal time intervals $t$ (the Hahn spin echo corresponds to $n=1$ ), allows measurements of even more interesting response functions. The corresponding overlap function is given by

$$
\left\langle\hat{S}_{x, n}(t)\right\rangle=\operatorname{Re}\left[S_{n}(t)\right], \quad S_{n}(t)=\left\langle\psi_{0}\left|\hat{U}_{2}^{-n} \hat{U}_{1}^{n}\right| \psi_{0}\right\rangle
$$

The long-time asymptotic behavior of the $n$th response, calculated in a similar fashion to the case of the Hahn spin echo, is characterized by the power-law decay:

$$
S_{n}(t) \propto t^{-\alpha_{n} \delta_{F}^{2} / \pi^{2}}, \quad \alpha_{n}=4 n-1 .
$$

Thus, by increasing the number of pulses $n$, the exponent can be strongly enhanced. This should facilitate the observation of the power-law decay in the spin-echo

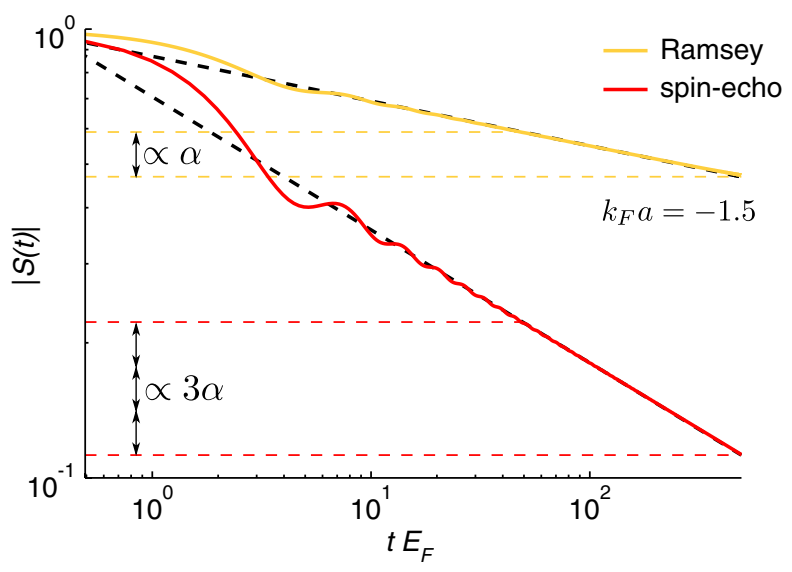

FIG. 6. The spin-echo response, Eq. (19), of the Fermi gas. At long times, it is characterized by a power-law decay, Eq. (20), with an exponent that is 3 times larger than that of the standard OC. 

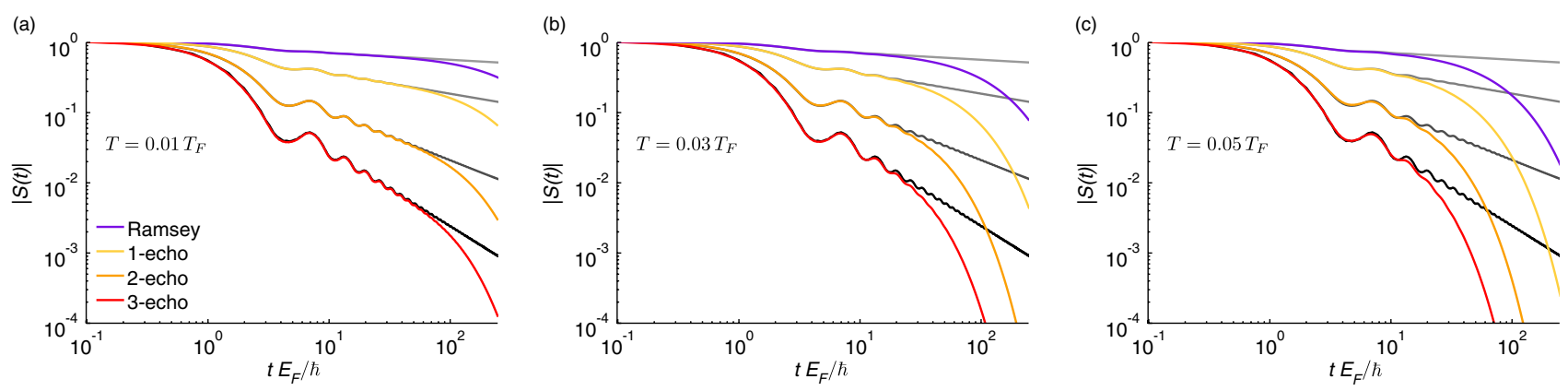

FIG. 7. The generalized spin-echo response, Eq. (21), of the Fermi gas for $n=1,2,3$ compared to the Ramsey interference. At long times, the generalized spin-echo response is characterized by a power-law decay, Eq. (22), with an exponent (4n-1) times larger than that of the standard OC. The gray lines correspond to $T=0$, while the colored lines are for finite temperatures (a) $T=0.01 T_{F}$, (b) $T=0.03 T_{F}$, and (c) $T=0.05 T_{F}$. The scattering length is $k_{F} a=-1.5$ in all cases. The power-law decay of the spin-echo response gives way to a faster exponential decay at times $t \gtrsim \hbar / T$.

experiments. The universal spin-echo responses for different values of $n$, calculated numerically, are illustrated in Fig. 7 along with Ramsey-interference results. The asymptotic form at large times agrees with the analytic formula given in Eq. (22).

Experiments are always done at low, but finite temperature; therefore, it is important to understand the effect of nonzero temperature $T$ on the spin-echo response. Within our approach, the finite-temperature response is found by calculating the corresponding determinants with the distribution functions $n(\varepsilon)=\frac{1}{\exp [(\varepsilon-\mu) / T]+1}$. The result, illustrated in Fig. 7, shows that the finite temperature does not affect the power-law behavior of the response at relatively short times $t \lesssim \hbar / T$; however, at longer times $t \gtrsim$ $\hbar / T$ the responses are characterized by a faster exponential decay. It is evident from Fig. 7 that the two most interesting features of the spin-echo response- the power-law decay and the oscillations with period $2 \pi \hbar / E_{F}$-survive up to experimentally accessible temperature $T=0.05 E_{F}$, and therefore they should be observable with current experimental means.

\section{MANIFESTATIONS OF THE OC IN ENERGY-COUNTING STATISTICS}

So far, we have considered two ways of observing the OC-the RF spectroscopy (energy domain), and the Ramsey interferometry, as well as spin-echo sequences (time domain). Both methods are based on studying the impurity properties. However, cold atomic systems also allow direct measurements of the Fermi-gas properties, e.g., measuring the individual occupation numbers of different states in the time-of-flight experiments, or with a single-atom quantum gas microscope [90,91]. Can one see traces of the OC by measuring properties of the Fermi gas following the sudden change of an impurity potential?

An obvious candidate quantity is the time-dependent density profile of the Fermi gas. We have studied the time-dependent density profile $\rho(t, r)$, finding that it does not carry signatures of the OC. This is because the overlap function involves a complicated sum of the $n$-particle-hole pair correlation functions, while the density operator can create at most one particle-hole pair. Thus, we are forced to consider the fluctuations rather than averages of physical quantities.

We note that the OC is formally related to the distribution function of the fluctuations of the total energy of the Fermi gas following an impurity quench. For simplicity, let us consider a situation where the impurity is initially in the interacting state $|\uparrow\rangle$, such that fermions are in the appropriate ground state $\left|\psi_{0}^{\prime}\right\rangle$; at some time, the impurity is suddenly flipped to the noninteracting state $|\downarrow\rangle$. Then, the characteristic function of the total energy distribution (with new Hamiltonian) is given by

$$
\chi(\lambda)=\left\langle\psi_{0}^{\prime}\left|e^{i \lambda \hat{H}_{0}}\right| \psi_{0}^{\prime}\right\rangle .
$$

Comparing with Eq. (2), we see that up to a phase factor, the characteristic function is identical to the overlap function, with parameter $\lambda$ playing the role of time. Thus, $\chi(\lambda)$ exhibits a power-law behavior at large $\lambda$ :

$$
\chi(\lambda) \propto \lambda^{-\delta_{F}^{2} / \pi^{2}} .
$$

This power-law asymptotic behavior, Eq. (23), gives rise to the universal power-law behavior of the probability distribution $P(E)$ at low energies $E \ll E_{F}$ :

$$
P(E)=\frac{1}{2 \pi} \int e^{-i \lambda E} \chi(\lambda) d \lambda \propto E^{\delta_{F}^{2} / \pi^{2}-1} .
$$

The unusual distribution function can be directly measured in the time-of-flight experiments (the total energy is given simply by the kinetic energy since in the final state the fermions are not interacting with the impurity). We note that time-of-flight experiments have already been used to measure the full energy of the system near the Feshbach resonance $[92,93]$. Furthermore, quantum-gas microscopes $[90,91]$ allow in situ measurements of energy deposited into 1D and 2D many-body systems following nonequilibrium 
evolution, including measurements of shot-to-shot fluctuations of the deposited energy. Quantum-gas microscopes therefore may provide a complementary technique for measuring the energy distribution function in quantumquench experiments. The power-law singularity in the energy fluctuations given in Eq. (24), similar to that found in the context of spin systems by Silva [77], provides a new experimentally accessible manifestation of the OC.

\section{THE NONEQUILIBRIUM OC AND QUANTUM TRANSPORT}

In this section, we schematically describe how the above ideas can be extended to study the nonequilibrium OC and quantum transport with cold atoms. An in-depth discussion will be given in a separate publication.

The orthogonality catastrophe is modified qualitatively when the Fermi gas is driven out of equilibrium [20,21]. In solid-state systems, such a situation occurs naturally when an impurity is coupled to two 1D leads with different chemical potentials. The impurity state controls the transmission between the two leads. From the mathematical point of view, the problem of the nonequilibrium OC can be reduced to a non-Abelian Riemann-Hilbert problem [20], which has not been solved in the general case. Progress has been made in the limit of long times, where the OC is characterized by the combination of a power-law decay with new exponents and weak exponential damping $[20,21]$.

We now argue that it is possible to study the nonequilibrium OC in cold atomic gases by generalizing the setup proposed above to the case of a multicomponent Fermi gas. For simplicity, let us consider a two-component gas, with pseudospin species $|u\rangle$ and $|d\rangle$. Our goal is to realize a situation in which two components are at different chemical potentials (playing the role of two leads), and an impurity that can scatter the fermions between two species. The first condition can be achieved by preparing an imbalanced Fermi gas, with different Fermi energies of two components, $E_{F}^{u}=E_{0}, E_{F}^{d}=E_{0}+\Delta \mu$. The second condition is more difficult to attain: An impurity's scattering matrix is diagonal in the $|u\rangle,|d\rangle$ basis, with phase shifts at the Fermi energy given by $\delta_{u, d}$. In the solid-state analogy, this corresponds to an impurity always fully reflecting electrons; effectively, this brings us back to two copies of the equilibrium OC.

To overcome this difficulty, we consider an application of a $\pi / 2$ pulse to the pseudospin of the host fermions. After that, the $|1\rangle=\frac{|u\rangle+|d\rangle}{\sqrt{2}}$ and $|2\rangle=\frac{|u\rangle-|d\rangle}{\sqrt{2}}$ states of fermions will be at different chemical potentials. Crucially, the impurity's scattering matrix is nondiagonal in the $|1\rangle,|2\rangle$ basis (it is obtained by a rotation of the $S$ matrix in the $|u\rangle,|d\rangle$ basis by matrix $\left.\frac{1+i \sigma_{y}}{\sqrt{2}}\right)$. The $|1\rangle,|2\rangle$ species plays the role of the electrons in the left and right leads in the mesoscopic experiment, with the impurity being characterized by a nontrivial scattering matrix. This is exactly the situation needed for realizing the nonequilibrium OC. Performing the Ramsey-interferometry or spinecho experiments on the impurity pseudospin then allows one to study the response of the nonequilibrium Fermi gas.

Looking beyond the OC, the analogy between the setup we have just considered and a quantum point contact with an impurity controlling the transmission through the quantum point contact suggests the possibility of studying the full-counting statistics of the charge transfer. Although theoretically charge-counting statistics has played an important role [94], its experimental studies in mesoscopic systems have been quite limited [79]. Simulating quantum transport in cold-atom experiments is also attractive because time-of-flight experiments allow energy-resolved measurements; this can be used to study correlations between the number of transmitted particles at different energies. It is worth mentioning that the possibility of measuring momentum-resolved occupation numbers has already been demonstrated experimentally; see, e.g., Ref. [93].

\section{CONCLUSIONS}

In conclusion, we studied the universal OC in cold atomic systems, and discussed the manifestations of the OC in the Ramsey and spin-echo interferometry, RF spectroscopy, as well as in energy-counting statistics. Beyond the equilibrium $\mathrm{OC}$, we have proposed a set of experiments which probe the dynamics and transport in nonequilibrium Fermi gases. This provides a useful connection between the cold-atom physics and mesoscopic physics.

The basic ingredients required for the experimental implementation of our proposal are the following: (a) a quantum-degenerate Bose-Fermi [29-36] or Fermi-Fermi [37-43] mixture, (b) the ability to trap one type of atom by a strong optical-lattice potential, (c) the control of the interaction strength between the impurity and host atom, which is achieved by changing the impurity hyperfine state, and (d) the ability to achieve temperatures that are low enough to observe the OC.

We emphasize that all four requirements are achievable with currently available techniques; thus, we expect that our proposal can be implemented in the near future. Below we describe some relevant experiments, which, we hope, will help to identify the systems that are most suitable for studying the OC.

(a) Various quantum-degenerate mixtures have been realized by multiple groups [29-43].

(b) Recently, the localization of minority atoms by an optical lattice was demonstrated with an imbalanced Bose-Bose mixture of ${ }^{87} \mathrm{Rb}$ and ${ }^{41} \mathrm{~K}$ atoms [95]. The localization of the impurity atoms at length scales of roughly $10 \%$ of the Fermi wavelength has been achieved at typical densities; thus, the impurity can be treated as pointlike, and one can neglect its 
excitations to the excited states of the trapping potentials, as in our analysis above.

(c) RF pulses have been used to switch between the hyperfine states of the impurity atoms, which interact differently with the host fermions [44]. This should enable the Ramsey and spin-echo-type experiments that reveal the OC. Furthermore, experiments with strongly imbalanced Fermi-Fermi mixtures, which addressed the polaron dynamics (mobile impurities) [44,46-49], demonstrated that the impurity-host atom interactions can be tuned in the full interaction range, from the strong-attractive to the strong-repulsive regime. This should allow for the exploration of different regimes of the OC, discussed above.

(d) In current experiments, temperatures as low as a few percent of the Fermi temperature can be achieved $[48,74,96]$. As follows from our analysis above, this should be sufficient to observe both the universal power-law decay as well as the oscillations with period $2 \pi \hbar / E_{F}$. In RF-absorption spectra, these oscillations result in a cusplike singularity at the Fermi energy $E_{F}$.

It is also worth noting that, for very short times, our results may be relevant to very heavy mobile impurities (with mass much larger than that of the host atoms). The OC will manifest itself, e.g., in the RF-spectroscopy experiments, which are commonly used to probe mixtures of cold atoms. Suitable mixtures with a large mass ratio are ${ }^{40} \mathrm{~K} /{ }^{41} \mathrm{~K}$ immersed in ${ }^{6} \mathrm{Li}[36,38-40,42]$ and ${ }^{173} \mathrm{Yb} /{ }^{174} \mathrm{Yb}$ immersed in ${ }^{6} \mathrm{Li}$ [43].

An alternative experimental route to accessing the nonequilibrium response of the Fermi gas to an impurity that is introduced suddenly is to create a local scattering potential by a narrow laser beam. This has been demonstrated in recent experiments [97,98]. While in such a setup one cannot perform the RF-absorption or Ramsey-interference experiments, an observation of the OC should be possible through energy-counting statistics; see Sec. IV. Furthermore, time-of-flight experiments would reveal the full distribution functions of scattering processes, providing new information about the nonequilibrium state of fermions.

Finally, we note that the ideas presented above are not limited to the case of free fermions; one particularly interesting extension concerns the case of 1D interacting fermions in an optical lattice. The OC in Luttinger liquids was predicted to exhibit strong deviations from the noninteracting case, showing new power laws that depend on the strength of interactions, universal power-law asymptotic behavior, as well as new scaling laws at intermediate times [99]. To access the possibility of observing these phenomena in a $1 \mathrm{D}$ optical lattices, we have carried out a numerical study [100] of interacting spinless fermions in a 1D lattice; we have found that the modifications of the OC in Luttinger liquids can be observed in finite optical lattices with only approximately 100 atoms, which put them within the reach of current experiments.

\section{ACKNOWLEDGMENTS}

We thank R. Grimm, C. Salomon, I. Zapata, and especially M. Zwierlein for many inspiring discussions. We acknowledge support from Harvard-MIT CUA, the NSF Grants No. DMR-07-05472 and No. DMR-1049082, the DARPA OLE program, AFOSR Quantum Simulation MURI, AFOSR MURI on Ultracold Molecules, the Austrian Science Fund (FWF) under Project No. P18551N16 (M. K.), the Austrian Marshall Plan Foundation (M. K.), the Welch Foundation, Grant No. C-1739, the A. P. Sloan Foundation (A. S. and A. I.), as well as the LANL Oppenheimer Fund (Y. N.).

\section{APPENDIX A: DETAILS ON THE NUMERICAL PROCEDURE}

In order to obtain the universal overlap function $S(t)$, we evaluate determinants of the type shown in Eq. (11) numerically. To this end, we consider a finite system confined in a sphere of radius $R$ whose eigenstates are in the absence and in the presence of the impurity:

$$
\begin{aligned}
& \psi_{n}(r)=\sqrt{\frac{2}{R}} \sin \left(k_{n} r\right), \quad k_{n} R=\pi n, \\
& \psi_{n}^{\prime}(r)=A_{n} \sqrt{\frac{2}{R}} \sin \left(k_{n}^{\prime} r+\delta_{n}\right), \quad k_{n}^{\prime} R+\delta_{n}=\pi n,
\end{aligned}
$$

respectively, where $A_{n}=1 / \sqrt{1+\frac{\sin 2 \delta_{n}}{2 k_{n}^{\prime} R}}$ and $\delta_{n}=$ $-\tan ^{-1}\left(k_{n}^{\prime} a\right)$. For $a>0$ there is also a bound state which must be treated separately. In principle, the evolution of the determinants requires, even at zero temperature, multiplications of infinite dimensional matrices. However, truncating the infinite number of intermediate states still allows us to evaluate the determinants with very high accuracy, since the OC is determined by low-energy physics. In order to obtain the universal overlap functions $S(t)$ from the finite system, we take a finite-size scaling by keeping the density constant while taking the system size to infinity. Importantly, in a finite-size system the overlap function $S(t)$ does not decay to zero but rather exhibits revivals after sufficiently long times characterized by the Fermi velocity and the system size - an additional aspect that should be observable in experiments.

We evaluate the universal RF spectra $A(\omega)$ from the Fourier transform of the overlap function $S(t)$. The RF spectra $A(\omega)$ exhibits power-law decays and band edges, which render a numerical evaluation of the Fourier transform extremely challenging, since $S(t)$ has to be known for 
extremely long times. To circumvent this problem, we calculate $S(t)$ exactly from Eq. (11) up to a certain time $t<$ $t^{*} \sim 500 \hbar E_{F}^{-1}$ and then continue $S(t)$ to longer times by fitting it to its asymptotic form, Eqs. (12) and (15), respectively. Even though, with that we have access to $S(t)$ for long times, the Fourier transform is still finite dimensional, which gives rise to wild oscillations at the edges of $A(\omega)$ known as Gibbs phenomenon. We reduce the Gibbs phenomenon by applying a Lanczos filter to the Fourier coefficients. With this procedure, we produce the universal RF-spectra shown in Fig. 2. Importantly, the positions of the band edges in the spectra match exactly the outcome of Eqs. (16) and (17).

\section{APPENDIX B: UNIVERSAL RADIO-FREQUENCY SPECTRA}

Here we provide the analytic formulas that describe the new feature in the absorption spectra $A(\omega)$ at frequency $\hbar \omega_{1}=\Delta E+E_{F}$ for $a<0$ and $\hbar \omega_{1}=\Delta E+E_{b}$ for $a>$ 0 , the origin of which is illustrated in Figs. 3(c) $(a<0)$ and 3(f) $(a>0)$. The configurations that lead to this nonanalyticity are given by the excitation of a fermion from the bottom of the band to the Fermi surface. The numerical results in Fig. 2 indicate that the nonanalytic feature develops into a stronger peak as unitarity is approached. This phenomenon, as well as the full structure of $A(\omega)$ at $k_{F}|a| \gg 1$, can be understood as a simple interplay between two-body physics happening near the bottom of the band, and physics of multiple particle-hole excitations being created at the Fermi surface.

In the time domain, the contributions from the Fermisurface excitations and the dynamics of the hole at the bottom of the band factorize. The former contributions manifest as the usual power laws with a unitary phase shift, while the latter can be simply evaluated within two-body theory; see [85] for details. In the frequency domain, the result, written as a convolution of the two terms corresponding to these two processes, reads

$$
\begin{aligned}
A(\omega) \approx & \frac{1.74 \pi}{\Gamma(1 / 4)\left(E_{F} / \hbar\right)^{1 / 4}} \int_{-\infty}^{\infty} \frac{d \varepsilon}{2 \pi} \theta\left(\omega-\omega_{1}+\frac{\varepsilon}{\hbar}\right) \\
& \times\left(\omega-\omega_{1}+\frac{\varepsilon}{\hbar}\right)^{-(3 / 4)} F(\varepsilon)+\mathrm{reg},
\end{aligned}
$$

where $F(\varepsilon)$ is the probability to excite a hole with energy $\varepsilon$ measured from the bottom of the band:

$$
F(\varepsilon)=\theta(\varepsilon) \frac{2 \sqrt{\hbar \varepsilon / E_{F}}}{\frac{\hbar^{2}}{2 m a^{2}}+\varepsilon}+\theta\left(k_{F} a\right) \frac{4 \pi \delta\left(\varepsilon-E_{b}\right)}{k_{F} a} .
$$

The numerical prefactor is evaluated similar to $C$, and function $F(\varepsilon)$ accounts for the existence of the bound state on the repulsive side. Terms denoted as "reg" account for a regular contribution at $\omega_{1}$ which is not singular at large $k_{F}|a|$.
Although for $0<\varepsilon \ll \hbar^{2} / 2 m a^{2}$, the function $F(\varepsilon)$ behaves as $\propto \sqrt{\varepsilon}$ and leads to a weak nonanalyticity, for $\hbar^{2} / 2 m a^{2} \ll \varepsilon$ it behaves as $\propto 1 / \sqrt{\varepsilon}$. Right at unitarity the scale $E_{b}$ disappears, and one obtains in $A(\omega)$ the divergence with the universal exponent $1 / 4$ and a universal shoulder ratio, see Eq. (18), which is valid for $\left|k_{F} a\right|=\infty$ and $\hbar\left|\omega-\omega_{1}\right| \ll E_{F}$. For large but finite negative $k_{F} a$, this peak gets smeared out at energies of the order of $\hbar^{2} / 2 m a^{2}$ from its maximal value, as is illustrated in the inset to Fig. 2(a). On the repulsive side, the true bound state with energy $E_{b}$ "pinches off" from the bottom of the band and leads to a threshold with an exponent $3 / 4$. Thus, for large but finite $k_{F} a>0$ the universal form of $A(\omega)$ near $\omega_{1}$ has a characteristic double-peak structure, as is seen in Fig. 2(b) for $k_{F} a=6.0$.

[1] Immanuel Bloch, Jean Dalibard, and Wilhelm Zwerger, Many-Body Physics with Ultracold Gases, Rev. Mod. Phys. 80, 885 (2008).

[2] Wolfgang Ketterle and Martin W. Zwierlein, in Proceedings of the International School of Physics Enrico Fermi, Course CLXIV, edited by M. Inguscio, W. Ketterle, and C. Salomon (IOS Press, Amsterdam, 2008).

[3] Markus Greiner, Olaf Mandel, Theodor W. Hänsch, and Immanuel Bloch, Collapse and Revival of the Matter Wave Field of a Bose-Einstein Condensate, Nature (London) 419, 51 (2002).

[4] H.-J. Miesner, D. M. Stamper-Kurn, J. Stenger, S. Inouye, A.P. Chikkatur, and W. Ketterle, Observation of Metastable States in Spinor Bose-Einstein Condensates, Phys. Rev. Lett. 82, 2228 (1999).

[5] L. E. Sadler, J. M. Higbie, S. R. Leslie, M. Vengalattore, and D.M. Stamper-Kurn, Spontaneous Symmetry Breaking in a Quenched Ferromagnetic Spinor BoseEinstein Condensate, Nature (London) 443, 312 (2006).

[6] Niels Strohmaier, Daniel Greif, Robert Jördens, Leticia Tarruell, Henning Moritz, Tilman Esslinger, Rajdeep Sensarma, David Pekker, Ehud Altman, and Eugene Demler, Observation of Elastic Doublon Decay in the Fermi-Hubbard Model, Phys. Rev. Lett. 104, 080401 (2010).

[7] Ulrich Schneider, Lucia Hackermüller, Jens Philipp Ronzheimer, Sebastian Will, Simon Braun, Thorsten Best, Immanuel Bloch, Eugene Demler, Stephan Mandt, David Rasch, and Achim Rosch, Fermionic Transport and Out-of-Equilibrium Dynamics in a Homogeneous Hubbard Model with Ultracold Atoms, Nat. Phys. 8, 213 (2012).

[8] Y. Shin, M. Saba, T. A. Pasquini, W. Ketterle, D. E. Pritchard, and A. E. Leanhardt, Atom Interferometry with Bose-Einstein Condensates in a Double-Well Potential, Phys. Rev. Lett. 92, 050405 (2004).

[9] S. Hofferberth, I. Lesanovsky, B. Fischer, T. Schumm, and J. Schmiedmayer, Non-Equilibrium Coherence Dynamics in One-Dimensional Bose Gases, Nature (London) 449, 324 (2007). 
[10] S. Trotzky, P. Cheinet, S. Fölling, M. Feld, U. Schnorrberger, A. M Rey, A. Polkovnikov, E. A Demler, M. D Lukin, and I. Bloch, Time-Resolved Observation and Control of Superexchange Interactions with Ultracold Atoms in Optical Lattices, Science 319, 295 (2008).

[11] Ming-Shien Chang, Qishu Qin, Wenxian Zhang, Li You, and Michael S. Chapman, Coherent Spinor Dynamics in a Spin-1 Bose-Condensate, Nat. Phys. 1, 111 (2005).

[12] Jasper Simon Krauser, Jannes Heinze, Nick Fläschner, Sören Götze, Christoph Becker, and Klaus Sengstock, Coherent Multi-Flavour Spin Dynamics in a Fermionic Quantum Gas, Nat. Phys. 8, 813 (2012).

[13] Toshiya Kinoshita, Trevor Wenger, and David S. Weiss, $A$ Quantum Newton's Cradle, Nature (London) 440, 900 (2006).

[14] S. Trotzky, Y-A. Chen, A. Flesch, I. P. McCulloch, U. Schollwöck, J. Eisert, and I. Bloch, Probing the Relaxation Towards Equilibrium in an Isolated Strongly Correlated One-Dimensional Bose Gas, Nat. Phys. 8, 325 (2012).

[15] Christian Sanner, Edward J. Su, Wujie Huang, Aviv Keshet, Jonathon Gillen, and Wolfgang Ketterle, Correlations and Pair Formation in a Repulsively Interacting Fermi Gas, Phys. Rev. Lett. 108, 240404 (2012).

[16] Gerald D. Mahan, Many Particle Physics (Kluwer, New York, 2000), 3rd ed.

[17] G. Yuval and P. W. Anderson, Exact Results for the Kondo Problem: One-body Theory and Extension to Finite Temperature, Phys. Rev. B 1, 1522 (1970).

[18] K. A. Matveev and A.I. Larkin, Interaction-Induced Threshold Singularities in Tunneling via Localized Levels, Phys. Rev. B 46, 15337 (1992).

[19] A. K. Geim, P. C. Main, N. La Scala, L. Eaves, T. J. Foster, P. H. Beton, J. W. Sakai, F. W. Sheard, M. Henini, G. Hill, and M.A. Pate, Fermi-Edge Singularity in Resonant Tunneling, Phys. Rev. Lett. 72, 2061 (1994).

[20] N. d'Ambrumenil and B. Muzykantskii, Fermi Gas Response to Time-Dependent Perturbations, Phys. Rev. B 71, 045326 (2005).

[21] D. A. Abanin and L.S. Levitov, Fermi-Edge Resonance and Tunneling in Nonequilibrium Electron Gas, Phys. Rev. Lett. 94, 186803 (2005).

[22] D. A. Abanin and L.S. Levitov, Tunable Fermi-Edge Resonance in an Open Quantum Dot, Phys. Rev. Lett. 93, 126802 (2004).

[23] Martina Hentschel, Denis Ullmo, and Harold U. Baranger, Fermi Edge Singularities in the Mesoscopic Regime: Anderson Orthogonality Catastrophe, Phys. Rev. B 72, 035310 (2005).

[24] M. Pustilnik, M. Khodas, A. Kamenev, and L. I. Glazman, Dynamic Response of One-Dimensional Interacting Fermions, Phys. Rev. Lett. 96, 196405 (2006); Rodrigo G. Pereira, Steven R. White, and Ian Affleck, Exact Edge Singularities and Dynamical Correlations in Spin-1/2 Chains, ibid. 100, 027206 (2008); Spectral Function of Spinless Fermions on a One-Dimensional Lattice, Phys. Rev. B 79, 165113 (2009).

[25] Adilet Imambekov and Leonid I. Glazman, Universal Theory of Nonlinear Luttinger Liquids, Science 323, 228
(2009); Phenomenology of One-Dimensional Quantum Liquids Beyond the Low-Energy Limit, Phys. Rev. Lett. 102, 126405 (2009).

[26] Adilet Imambekov, Thomas L. Schmidt, and Leonid I. Glazman, One-Dimensional Quantum Liquids: Beyond the Luttinger Liquid Paradigm, Rev. Mod. Phys. 84, 1253 (2012).

[27] Achim Rosch, Quantum-Coherent Transport of a Heavy Particle in a Fermionic Bath, Adv. Phys. 48, 295 (1999).

[28] Yuli V. Nazarov and Yaroslav M. Blanter, Quantum Transport: Introduction to Nanoscience (Cambridge University Press, Cambridge, 2009), 1st ed.

[29] F. Schreck, L. Khaykovich, K. L. Corwin, G. Ferrari, T. Bourdel, J. Cubizolles, and C. Salomon, Quasipure BoseEinstein Condensate Immersed in a Fermi Sea, Phys. Rev. Lett. 87, 080403 (2001).

[30] S. Ospelkaus, C. Ospelkaus, O. Wille, M. Succo, P. Ernst, K. Sengstock, and K. Bongs, Localization of Bosonic Atoms by Fermionic Impurities in a Three-Dimensional Optical Lattice, Phys. Rev. Lett. 96, 180403 (2006).

[31] Kenneth Günter, Thilo Stöferle, Henning Moritz, Michael Köhl, and Tilman Esslinger, Bose-Fermi Mixtures in a Three-Dimensional Optical Lattice, Phys. Rev. Lett. 96, 180402 (2006).

[32] M. Zaccanti, C. D'Errico, F. Ferlaino, G. Roati, M. Inguscio, and G. Modugno, Control of the Interaction in a Fermi-Bose Mixture, Phys. Rev. A 74, 041605 (2006).

[33] Th. Best, S. Will, U. Schneider, L. Hackermüller, D. van Oosten, I. Bloch, and D.-S. Lühmann, Role of Interactions in ${ }^{87} \mathrm{Rb}-{ }^{40} \mathrm{~K}$ Bose-Fermi Mixtures in a 3 D Optical Lattice, Phys. Rev. Lett. 102, 030408 (2009).

[34] Takeshi Fukuhara, Seiji Sugawa, Yosuke Takasu, and Yoshiro Takahashi, All-Optical Formation of Quantum Degenerate Mixtures, Phys. Rev. A 79, 021601 (2009).

[35] Meng Khoon Tey, Simon Stellmer, Rudolf Grimm, and Florian Schreck, Double-Degenerate Bose-Fermi Mixture of Strontium, Phys. Rev. A 82, 011608 (2010).

[36] Cheng-Hsun Wu, Ibon Santiago, Jee Woo Park, Peyman Ahmadi, and Martin W. Zwierlein, Strongly Interacting Isotopic Bose-Fermi Mixture Immersed in a Fermi Sea, Phys. Rev. A 84, 011601(R) (2011).

[37] Martin W. Zwierlein, André Schirotzek, Christian H. Schunck, and Wolfgang Ketterle, Fermionic Superfluidity with Imbalanced Spin Populations, Science 311, 492 (2006).

[38] M. Taglieber, A.-C. Voigt, T. Aoki, T. W. Hänsch, and K. Dieckmann, Quantum Degenerate Two-Species FermiFermi Mixture Coexisting with a Bose-Einstein Condensate, Phys. Rev. Lett. 100, 010401 (2008).

[39] E. Wille, F.M. Spiegelhalder, G. Kerner, D. Naik, A. Trenkwalder, G. Hendl, F. Schreck, R. Grimm, T. G. Tiecke, J. T. M. Walraven, S. J. J. M. F. Kokkelmans, E. Tiesinga, and P.S. Julienne, Exploring an Ultracold Fermi-Fermi Mixture: Interspecies Feshbach Resonances and Scattering Properties of ${ }^{6} \mathrm{Li}$ and ${ }^{40} \mathrm{~K}$, Phys. Rev. Lett. 100, 053201 (2008).

[40] T. G. Tiecke, M. R. Goosen, A. Ludewig, S. D. Gensemer, S. Kraft, S. J. J. M. F. Kokkelmans, and J. T. M. Walraven, Broad Feshbach Resonance in the ${ }^{6} \mathrm{Li}^{-}{ }^{40} \mathrm{~K}$ Mixture, Phys. Rev. Lett. 104, 053202 (2010). 
[41] Yean-an Liao, Ann Sophie C. Rittner, Tobias Paprotta, Wenhui Li, Guthrie B. Partridge, Randall G. Hulet, Stefan K. Baur, and Erich J. Mueller, Spin-Imbalance in a OneDimensional Fermi Gas, Nature (London) 467, 567 (2010).

[42] A. Trenkwalder, C. Kohstall, M. Zaccanti, D. Naik, A. I. Sidorov, F. Schreck, and R. Grimm, Hydrodynamic Expansion of a Strongly Interacting Fermi-Fermi Mixture, Phys. Rev. Lett. 106, 115304 (2011).

[43] Hideaki Hara, Yosuke Takasu, Yoshifumi Yamaoka, John M. Doyle, and Yoshiro Takahashi, Quantum Degenerate Mixtures of Alkali and Alkaline-Earth-Like Atoms, Phys. Rev. Lett. 106, 205304 (2011).

[44] C. Kohstall, M. Zaccanti, M. Jag, A. Trenkwalder, P. Massignan, G. M. Bruun, F. Schreck, and R. Grimm, Metastability and Coherence of Repulsive Polarons in a Strongly Interacting Fermi Mixture, Nature (London) 485, 615 (2012).

[45] E. Tiesinga, C. J. Williams, F. H. Mies, and P. S. Julienne, Interacting Atoms under Strong Quantum Confinement, Phys. Rev. A 61, 063416 (2000).

[46] André Schirotzek, Cheng-Hsun Wu, Ariel Sommer, and Martin W. Zwierlein, Observation of Fermi Polarons in a Tunable Fermi Liquid of Ultracold Atoms, Phys. Rev. Lett. 102, 230402 (2009).

[47] S. Nascimbène, N. Navon, K. J. Jiang, L. Tarruell, M. Teichmann, J. McKeever, F. Chevy, and C. Salomon, Collective Oscillations of an Imbalanced Fermi Gas: Axial Compression Modes and Polaron Effective Mass, Phys. Rev. Lett. 103, 170402 (2009).

[48] N. Navon, S. Nascimbène, F. Chevy, and C. Salomon, The Equation of State of a Low-Temperature Fermi Gas with Tunable Interactions, Science 328, 729 (2010).

[49] Marco Koschorreck, Daniel Pertot, Enrico Vogt, Bernd Fröhlich, Michael Feld, and Michael Köhl, Attractive and Repulsive Fermi Polarons in Two Dimensions, Nature (London) 485, 619 (2012).

[50] We take the energy difference between discrete levels of the impurity in a confining potential to be much larger than the Fermi energy of the host atoms so that we can neglect the scattering of the impurity atom into excited states.

[51] G. D. Mahan, Excitons in Metals: Infinite Hole Mass, Phys. Rev. 163, 612 (1967).

[52] P. Nozières and C.T. De Dominicis, Singularities in the X-Ray Absorption and Emission of Metals. III. OneBody Theory Exact Solution, Phys. Rev. 178, 1097 (1969).

[53] P. W. Anderson, Infrared Catastrophe in Fermi Gases with Local Scattering Potentials, Phys. Rev. Lett. 18, 1049 (1967).

[54] Alexander O. Gogolin, Alexander A. Nersesyan, and Alexei M. Tsvelik, Bosonization and Strongly Correlated Systems (Cambridge University Press, Cambridge, 2004).

[55] M. Combescot and P. Nozières, Infrared Catastrophy and Excitions in the X-Ray Spectra of Metals, J. Phys. (Paris) 32, 913 (1971).

[56] K. Ohtaka and Y. Tanabe, Theory of the Soft-X-Ray Edge Problem in Simple Metals: Historical Survey and Recent Developments, Rev. Mod. Phys. 62, 929 (1990).
[57] V. V. Mkhitaryan and M. E. Raikh, Fermi-Edge Singularity in the Vicinity of the Resonant Scattering Condition, Phys. Rev. Lett. 106, 197003 (2011).

[58] It is important to point out that the scattering length $a$ characterizing the scattering of the host fermions on the localized impurity is not the same as the scattering length of two free atoms $[59,60]$. In this paper, we take the energy difference between discrete levels of the impurity in a confining potential to be much larger than the Fermi energy of the host atoms so that we can neglect the scattering of the impurity atom into excited states; then the scattering amplitude has the canonical form, which is given in Eq. (3). In principle, when the Fermi energy becomes comparable to the energy splitting between discrete levels $\omega_{c}$ in the parabolic potential confining the impurity, there will be an additional dependence of $f(E)$ on the $E / \omega_{c}$, discussed in [59]; therefore, all response functions will also depend on $E_{F} / \omega_{c}$. Our discussion can be extended to find these dependencies.

[59] Yusuke Nishida and Shina Tan, Confinement-Induced p-Wave Resonances from s-Wave Interactions, Phys. Rev. A 82, 062713 (2010).

[60] Eric Vernier, David Pekker, Martin W. Zwierlein, and Eugene Demler, Bound States of a Localized Magnetic Impurity in a Superfluid of Paired Ultracold Fermions, Phys. Rev. A 83, 033619 (2011).

[61] T. Schumm, S. Hofferberth, L. M. Andersson, S. Wildermuth, S. Groth, I. Bar-Joseph, J. Schmiedmayer, and P. Krüger, Matter-Wave Interferometry in a Double Well on an Atom Chip, Nat. Phys. 1, 57 (2005).

[62] C. Gross, T. Zibold, E. Nicklas, J. Estève, and M. K. Oberthaler, Nonlinear Atom Interferometer Surpasses Classical Precision Limit, Nature (London) 464, 1165 (2010).

[63] Takuya Kitagawa, Susanne Pielawa, Adilet Imambekov, Jörg Schmiedmayer, Vladimir Gritsev, and Eugene Demler, Ramsey Interference in One-Dimensional Systems: The Full Distribution Function of Fringe Contrast as a Probe of Many-Body Dynamics, Phys. Rev. Lett. 104, 255302 (2010).

[64] Artur Widera, Stefan Trotzky, Patrick Cheinet, Simon Fölling, Fabrice Gerbier, Immanuel Bloch, Vladimir Gritsev, Mikhail D. Lukin, and Eugene Demler, Quantum Spin Dynamics of Mode-Squeezed Luttinger Liquids in Two-Component Atomic Gases, Phys. Rev. Lett. 100, 140401 (2008).

[65] Michael Gring, Maximilian Kuhnert, Tim Langen, Takuya Kitagawa, Bernhard Rauer, Matthias Schreitl, Igor Mazets, David A. Smith, Eugene Demler, and Jörg Schmiedmayer, Relaxation Dynamics and PreThermalization in an Isolated Quantum System, Science 337, 1318 (2012).

[66] J. Goold, T. Fogarty, N. Lo Gullo, M. Paternostro, and Th. Busch, Orthogonality Catastrophe as a Consequence of Qubit Embedding in an Ultracold Fermi Gas, Phys. Rev. A 84, 063632 (2011).

[67] By changing the phase $\varphi$ of the closing $\pi / 2$ pulse we can obtain $\operatorname{Re}\left[e^{i \varphi} S(t)\right]$.

[68] We note that the idea that the interferometry of a single atom entangled with a many-body system can be employed to probe dynamics of the many-body system, has 
been discussed previously in a different context in Ref. [69].

[69] A. Micheli, A. J. Daley, D. Jaksch, and P. Zoller, Single Atom Transistor in a $1 D$ Optical Lattice, Phys. Rev. Lett. 93, 140408 (2004).

[70] D. M. Eagles, Possible Pairing without Superconductivity at Low Carrier Concentrations in Bulk and Thin-Film Superconducting Semiconductors, Phys. Rev. 186, 456 (1969); P. Nozières and S. Schmitt-Rink, Bose Condensation in an Attractive Fermion Gas: From Weak to Strong Coupling Superconductivity, J. Low Temp. Phys. 59, 195 (1985); A. Leggett, in Modern Trends in the Theory of Condensed Matter, edited by Andrzej Pekalski and Jerzy Przystawa (Springer, Berlin, 1980), Vol. 115, pp. 13; Y. Ohashi and A. Griffin, Superfluid Transition Temperature in a Trapped Gas of Fermi Atoms with a Feshbach Resonance, Phys. Rev. A 67, 033603 (2003); BCS-BEC Crossover in a Gas of Fermi Atoms with a Feshbach Resonance, Phys. Rev. Lett. 89, 130402 (2002).Qijin Chen, Jelena Stajic, Shina Tan, and K. Levin, BCS-BEC Crossover: From High Temperature Superconductors to Ultracold Superfluids, Phys. Rep. 412, 1 (2005).

[71] Jean-Philippe Brantut, Jakob Meineke, David Stadler, Sebastian Krinner, and Tilman Esslinger, Conduction of Ultracold Fermions through a Mesoscopic Channel, Science 337, 1069 (2012).

[72] M. J. Davis, P. B. Blakie, A. H. van Amerongen, N. J. van Druten, and K. V. Kheruntsyan, Yang-Yang Thermometry and Momentum Distribution of a Trapped OneDimensional Bose Gas, Phys. Rev. A 85, 031604 (2012).

[73] Toshiya Kinoshita, Trevor Wenger, and David S. Weiss, Observation of a One-Dimensional Tonks-Girardeau Gas, Science 305, 1125 (2004).

[74] Mark J. H. Ku, Ariel T. Sommer, Lawrence W. Cheuk, and Martin W. Zwierlein, Revealing the Superfluid Lambda Transition in the Universal Thermodynamics of a Unitary Fermi Gas, Science 335, 563 (2012).

[75] Simon Fölling, Fabrice Gerbier, Artur Widera, Olaf Mandel, Tatjana Gericke, and Immanuel Bloch, Spatial Quantum Noise Interferometry in Expanding Ultracold Atom Clouds, Nature (London) 434, 481 (2005); Christian Sanner, Edward J. Su, Aviv Keshet, Ralf Gommers, Yong-il Shin, Wujie Huang, and Wolfgang Ketterle, Suppression of Density Fluctuations in a Quantum Degenerate Fermi Gas, Phys. Rev. Lett. 105, 040402 (2010); Torben Müller, Bruno Zimmermann, Jakob Meineke, Jean-Philippe Brantut, Tilman Esslinger, and Henning Moritz, Local Observation of Antibunching in a Trapped Fermi Gas, Phys. Rev. Lett. 105, 040401 (2010); A. Perrin, R. Bücker, S. Manz, T. Betz, C. Koller, T. Plisson, T. Schumm, and J. Schmiedmayer, Hanbury Brown and Twiss Correlations across the Bose-Einstein Condensation Threshold, Nat. Phys. 8, 195 (2012).

[76] S. Hofferberth, I. Lesanovsky, T. Schumm, A. Imambekov, V. Gritsev, E. Demler, and J. Schmiedmayer, Probing Quantum and Thermal Noise in an Interacting ManyBody System, Nat. Phys. 4, 489 (2008).

[77] Alessandro Silva, Statistics of the Work Done on a Quantum Critical System by Quenching a Control Parameter, Phys. Rev. Lett. 101, 120603 (2008).
[78] Pietro Smacchia and Alessandro Silva, Universal Energy Distribution of Quasiparticles Emitted in a Local TimeDependent Quench, Phys. Rev. Lett. 109, 037202 (2012).

[79] B. Reulet, J. Senzier, and D. E. Prober, Environmental Effects in the Third Moment of Voltage Fluctuations in a Tunnel Junction, Phys. Rev. Lett. 91, 196601 (2003); Yu. Bomze, G. Gershon, D. Shovkun, L.S. Levitov, and M. Reznikov, Measurement of Counting Statistics of Electron Transport in a Tunnel Junction, Phys. Rev. Lett. 95, 176601 (2005); S. Gustavsson, R. Leturcq, B. Simovič, R. Schleser, T. Ihn, P. Studerus, K. Ensslin, D. C. Driscoll, and A.C. Gossard, Counting Statistics of Single Electron Transport in a Quantum Dot, Phys. Rev. Lett. 96, 076605 (2006).

[80] I. Klich, in Quantum Noise in Mesoscopic Systems, edited by Yu. V. Nazarov (Kluwer, Dordrecht, 2003), pp. 397.

[81] I. V Protopopov, D. B Gutman, and A. D Mirlin, ManyParticle Correlations in a Non-Equilibrium Luttinger Liquid, J. Stat. Mech. (2011) P11001.

[82] Ian Aeck, Boundary Condition Changing Operations in Conformal Field Theory and Condensed Matter Physics, Nucl. Phys. B, Proc. Suppl. 58, 35 (1997).

[83] We note that these prefactors can also be investigated from following the method of Ref. [84]. The details of such a procedure, which leads to fully analytical results, will be published elsewhere [85].

[84] Aditya Shashi, Leonid I. Glazman, Jean-Sébastien Caux, and Adilet Imambekov, Nonuniversal Prefactors in the Correlation Functions of One-Dimensional Quantum Liquids, Phys. Rev. B 84, 045408 (2011); Aditya Shashi, Milosz Panfil, Jean-Sébastien Caux, and Adilet Imambekov, Exact Prefactors in Static and Dynamic Correlation Functions of One-Dimensional Quantum Integrable Models: Applications to the CalogeroSutherland, Lieb-Liniger, and XXZ Models, Phys. Rev. B 85, 155136 (2012).

[85] A. Shashi et al. (unpublished).

[86] Shina Tan, Energetics of a Strongly Correlated Fermi Gas, Ann. Phys. (Amsterdam) 323, 2952 (2008); Large Momentum Part of a Strongly Correlated Fermi Gas 323, 2971 (2008); Generalized Virial Theorem and Pressure Relation for a Strongly Correlated Fermi Gas323, 2987 (2008).

[87] Eric Braaten, Daekyoung Kang, and Lucas Platter, Universal Relations for a Strongly Interacting Fermi Gas near a Feshbach Resonance, Phys. Rev. A 78, 053606 (2008); Eric Braaten and Lucas Platter, Exact Relations for a Strongly Interacting Fermi Gas from the Operator Product Expansion, Phys. Rev. Lett. 100, 205301 (2008); R. Haussmann, M. Punk, and W. Zwerger, Spectral Functions and rf Response of Ultracold Fermionic Atoms, Phys. Rev. A 80, 063612 (2009); R. Combescot, F. Alzetto, and X. Leyronas, Particle Distribution Tail and Related Energy Formula, Phys. Rev. A 79, 053640 (2009); F. Werner, L. Tarruell, and Y. Castin, Number of Closed-Channel Molecules in the BEC-BCS Crossover, Eur. Phys. J. B 68, 401 (2009); William Schneider and Mohit Randeria, Universal ShortDistance Structure of the Single-Particle Spectral Function of Dilute Fermi Gases, Phys. Rev. A 81, 021601 (2010); Eric Braaten, Daekyoung Kang, and 
Lucas Platter, Universal Relations for Identical Bosons from Three-Body Physics, Phys. Rev. Lett. 106, 153005 (2011); Shizhong Zhang and Anthony J. Leggett, Universal Properties of the Ultracold Fermi Gas, Phys. Rev. A 79, 023601 (2009).

[88] E. D. Kuhnle, H. Hu, X.-J. Liu, P. Dyke, M. Mark, P. D. Drummond, P. Hannaford, and C.J. Vale, Universal Behavior of Pair Correlations in a Strongly Interacting Fermi Gas, Phys. Rev. Lett. 105, 070402 (2010); J. T. Stewart, J.P. Gaebler, T.E. Drake, and D.S. Jin, Verification of Universal Relations in a Strongly Interacting Fermi Gas, Phys. Rev. Lett. 104, 235301 (2010); R. J. Wild, P. Makotyn, J. M. Pino, E. A. Cornell, and D. S. Jin, Measurements of Tan's Contact in an Atomic Bose-Einstein Condensate, Phys. Rev. Lett. 108, 145305 (2012).

[89] Philippe Nozières, Theory Of Interacting Fermi Systems (Addison-Wesley, Reading, MA, 1997).

[90] Waseem S. Bakr, Jonathon I. Gillen, Amy Peng, Simon Fölling, and Markus Greiner, A Quantum Gas Microscope for Detecting Single Atoms in a Hubbard-Regime Optical Lattice, Nature (London) 462, 74 (2009).

[91] Jacob F. Sherson, Christof Weitenberg, Manuel Endres, Marc Cheneau, Immanuel Bloch, and Stefan Kuhr, SingleAtom-Resolved Fluorescence Imaging of an Atomic Mott Insulator, Nature (London) 467, 68 (2010).

[92] T. Bourdel, J. Cubizolles, L. Khaykovich, K. M. F. Magalhaes, S. J. J. M. F. Kokkelmans, G. V. Shlyapnikov, and C. Salomon, Measurement of Interaction Energy near a Feshbach Resonance in a 6Li Fermi Gas, Phys. Rev. Lett. 91, 020402 (2003).
[93] J. T. Stewart, J.P. Gaebler, T.E. Drake, and D. S. Jin, Verification of Universal Relations in a Strongly Interacting Fermi Gas, Phys. Rev. Lett. 104, 235301 (2010).

[94] Leonid S. Levitov, Hyunwoo Lee, and Gordey B. Lesovik, Electron Counting Statistics and Coherent States of Electric Current, J. Math. Phys. (N.Y.) 37, 4845 (1996).

[95] J. Catani, G. Lamporesi, D. Naik, M. Gring, M. Inguscio, F. Minardi, A. Kantian, and T. Giamarchi, Quantum Dynamics of Impurities in a One-Dimensional Bose Gas, Phys. Rev. A 85, 023623 (2012).

[96] G. B. Partridge, Wenhui Li, Y. A. Liao, R. G. Hulet, M. Haque, and H. T.C. Stoof, Deformation of a Trapped Fermi Gas with Unequal Spin Populations, Phys. Rev. Lett. 97, 190407 (2006).

[97] Christof Weitenberg, Manuel Endres, Jacob F. Sherson, Marc Cheneau, Peter Schau, Takeshi Fukuhara, Immanuel Bloch, and Stefan Kuhr, Single-Spin Addressing in an Atomic Mott Insulator, Nature (London) 471, 319 (2011).

[98] Rémi Desbuquois, Lauriane Chomaz, Tarik Yefsah, Julian Léonard, Jérôme Beugnon, Christof Weitenberg, and Jean Dalibard, Superfluid Behaviour of a Two-Dimensional Bose Gas, Nat. Phys. 8, 645 (2012).

[99] Alexander O. Gogolin, Local Time-Dependent Perturbation in Luttinger Liquid, Phys. Rev. Lett. 71, 2995 (1993); N. V. Prokof'ev, Fermi-Edge Singularity with Backscattering in the Luttinger-Liquid Model, Phys. Rev. B 49, 2148 (1994); V. Meden, P. Schmitteckert, and Nic Shannon, Orthogonality Catastrophe in a OneDimensional System of Correlated Electrons, Phys. Rev. B 57, 8878 (1998).

[100] M. Knap et al. (unpublished). 